B. R. Choe and H. Yi

Nagoya Math. J.

Vol. 151 (1998), 51-89

\title{
REPRESENTATIONS AND INTERPOLATIONS OF HARMONIC BERGMAN FUNCTIONS ON HALF-SPACES
}

\author{
BOO RIM CHOE ${ }^{1}$ AND HEUNGSU YI $^{2}$
}

\begin{abstract}
On the setting of the half-space of the euclidean $n$-space, we prove representation theorems and interpolation theorems for harmonic Bergman functions in a constructive way. We also consider the harmonic (little) Bloch spaces as limiting spaces. Our results show that well-known phenomena for holomorphic cases continue to hold. Our proofs of representation theorems also yield a uniqueness theorem for harmonic Bergman functions. As an application of interpolation theorems, we give a distance estimate to the harmonic little Bloch space. In the course of the proofs, pseudohyperbolic balls are used as substitutes for Bergman metric balls in the holomorphic case.
\end{abstract}

\section{$\S 1$. Introduction}

Let $H=H_{n}(n \geq 2)$ denote the upper half-space $\mathbb{R}^{n-1} \times \mathbb{R}_{+}$where $\mathbb{R}_{+}$ denotes the set of all positive real numbers. For $1 \leq p<\infty$, we will write $b^{p}$ for the harmonic Bergman space consisting of all harmonic functions $u$ on $H$ such that

$$
\|u\|_{p}=\left\{\int_{H}|u|^{p} d V\right\}^{1 / p}<\infty
$$

where $V$ denotes the volume measure on $H$. The space $b^{p}$ turns out to be a closed subspace of $L^{p}$, the Lebesgue space on $H$, and thus $b^{p}$ is a Banach space. In particular, $b^{2}$ is a Hilbert space. Hence, there is a unique Hilbert space orthogonal projection $R: L^{2} \rightarrow b^{2}$, which is called the harmonic Bergman projection. It is known (see Theorem 8.22 of [3]) that this harmonic Bergman projection can be realized as an integral operator against the harmonic Bergman kernel $R(z, w)$. See Section 2 .

In their recent paper [8], Ramey and Yi have studied these harmonic Bergman spaces and have shown that many fundamental Bergman space

\footnotetext{
Received October 14, 1996.

${ }^{1}$ The first author was partially supported by KOSEF(95-0701-01-01-3), BSRI(97-1407) and GARC(97).

${ }^{2}$ The second author was partially supported by BSRI(97-1412).
} 
properties, which are well known in the holomorphic case, remain true. In this paper we continue to study further properties of these spaces. Here, we are concerned with two properties of harmonic Bergman functions. One property we are interested in is the representation of $b^{p}$-functions and the other is, its "dual" property, the interpolation by $b^{p}$-functions.

Roughly speaking, by the representation property, we mean the discrete version of the reproducing property of the harmonic Bergman kernel. That is, we mean the property of $b^{p}$-functions $u$ that can be represented as sums based on the harmonic Bergman kernel with weighted $l^{p}$-coefficients, which may take the following form:

$$
u(z)=\sum \lambda_{m} z_{m n}^{n(1-1 / p)} R\left(z, z_{m}\right) .
$$

Here, $z_{m n}$ denotes the last coordinate of $z_{m} \in H$. For example, the following is a special case of our representation results.

THEOREM 1.1. Suppose $1<p<\infty$. There exist a sequence $\left\{z_{m}\right\}$ of points in $H$ and a constant $C$ with the following properties: For $\left(\lambda_{m}\right) \in l^{p}$, the function $u$ defined by the series (1.1) belongs to $b^{p}$ with

$$
\int_{H}|u|^{p} d V \leq C \sum\left|\lambda_{m}\right|^{p} .
$$

Conversely, given $u \in b^{p}$, there exists a sequence $\left(\lambda_{m}\right) \in l^{p}$ such that (1.1) holds and

$$
\sum\left|\lambda_{m}\right|^{p} \leq C \int_{H}|u|^{p} d V .
$$

The corresponding theorem for the space $b^{1}$ is also available with a certain restriction. Note that this representation property amounts to the "onto" property of the operator $\left(\lambda_{m}\right) \mapsto u(z)$ from $l^{p}$ into $b^{p}$ defined by (1.1). By a consideration of the "onto" property of its adjoint operator, one is naturally led to the interpolation property. Roughly speaking, the interpolation property means that $l^{p}$ can be realized as a certain weighted sequence spaces formed by values of $b^{p}$-functions on a sequence of points in $H$. The following is the interpolation counterpart of Theorem 1.1, which is also a special case of our interpolation results.

THEOREM 1.2. Suppose $1 \leq p<\infty$. There exist a sequence $\left\{z_{m}\right\}$ of points in $H$ and a constant $C$ with the following properties: For $u \in b^{p}$, we have

$$
\sum z_{m n}^{n}\left|u\left(z_{m}\right)\right|^{p} \leq C \int_{H}|u|^{p} d V .
$$


Conversely, given $\left(\lambda_{m}\right) \in l^{p}$, there exists a function $u \in b^{p}$ such that $z_{m n}^{n / p} u\left(z_{m}\right)=\lambda_{m}$ for all $m$ and

$$
\int_{H}|u|^{p} d V \leq C \sum\left|\lambda_{m}\right|^{p}
$$

These two properties of holomorphic Bergman spaces were studied on various settings. See [5], [7] for the representation and [1], [9] for the interpolation. In [5], the representation properties of harmonic Bergman functions, as well as harmonic Bloch functions, are also proved on the unit ball of $\mathbb{R}^{n}$. The interpolation for holomorphic (little) Bloch functions were studied in [2], [4]. We will also consider the harmonic Bloch space $\widetilde{\mathcal{B}}$ and the harmonic little Bloch space $\widetilde{\mathcal{B}_{0}}$ on $H$ as limiting spaces of $b^{p}$. See Section 2 for definitions of these spaces. The models for our arguments are taken from those arguments in [5], [9] which are based on estimates of the Bergman kernel in a more constructive way. However, those arguments cannot be simply applied to the setting of the present paper. There are two fundamental reasons. First, as pointed out in [8], the unboundedness of $H$ causes certain difficulties especially when dealing with the spaces $b^{1}$ and $\widetilde{\mathcal{B}}$. For example, the kernel $R(z, w)$ is not integrable in each variable. Secondly, the kernel $R(z, w)$ has zeros, which causes difficulties in our estimates.

In Section 2 we list some basic properties related to the spaces $b^{p}, \widetilde{\mathcal{B}}$ and $\widetilde{\mathcal{B}}_{0}$.

In Section 3 we collect basic and computational lemmas which will be used repeatedly in later sections. Especially, we introduce a metric $\rho$ on $H$ which is very useful for our purposes. While euclidean balls with radii proportional to the distance from their centers to the boundary could be used as substitutes for Bergman metric balls in the holomorphic case, the $\rho$-balls play the role of Bergman metric balls and simplify a lot of our argument.

Section 4 is devoted to the proofs of the representation theorems for the space $b^{p}$, as well as the spaces $\widetilde{\mathcal{B}}$ and $\widetilde{\mathcal{B}}_{0}$. We consider here normal derivative versions of an arbitrary order. By a slight modification of our proof of normal derivative versions of the $b^{p}$-representation theorem, we prove that the $b^{p}$-norm is equivalent to the normal derivative lattice norm (see Proposition 4.8). This yields an unexpected result: If $a b^{p}$-function has vanishing normal derivatives along a "sufficiently dense" sequence, then it must vanish identically. This uniqueness theorem for $b^{p}$-functions seems 
new for harmonic Bergman functions. The analogue for harmonic Bloch functions is also proved (see Proposition 4.12).

Section 5 is devoted to the proofs of interpolation theorems for the space $b^{p}$, as well as the spaces $\widetilde{\mathcal{B}}$ and $\widetilde{\mathcal{B}}_{0}$. Using the $\widetilde{\mathcal{B}}_{0}$-interpolation theorem, we give an estimate for the distance between a given $\widetilde{\mathcal{B}}$-function and the space $\widetilde{\mathcal{B}}_{0}$, which might have further applications.

\section{$\S 2 . \quad$ Preliminaries}

In this section, we review some preliminary results from [3], [8]. Unless otherwise stated, the full range $1 \leq p<\infty$ is intended when discussing $b^{p}$.

By the mean value property and Jensen's inequality, one can easily verify that

$$
|u(z)|^{p} \leq \sigma_{n}^{-1} z_{n}^{-n}\|u\|_{p}^{p}
$$

holds for all $u \in b^{p}$ and $z \in H$. Here, we use the notation $\sigma_{n}$ for the volume of the unit ball of $\mathbb{R}^{n}$ and $z=\left(z^{\prime}, z_{n}\right) \in \mathbb{R}^{n-1} \times \mathbb{R}_{+}$for $z \in H$. It follows from inequality (2.1) that norm convergence in $b^{p}$ implies uniform convergence on compact subsets of $H$. Thus, $b^{p}$ is a Banach space and in particular $b^{2}$ is a Hilbert space. Inequality (2.1) also implies that, for each fixed $z \in H$, the map $z \mapsto u(z)$ is a bounded linear functional on $b^{2}$ and hence there exists a unique function $R(z, \cdot) \in b^{2}$ such that

$$
u(z)=\int_{H} u(w) \overline{R(z, w)} d w
$$

for all $u \in b^{2}$ where $d w$ also denotes the volume measure on $H$, which we will often write as $d z, d s$, etc. The function $R(z, w)$ is called the harmonic Bergman kernel for $b^{2}$. It is known that $R(z, w)=R(w, z)$ and that $R(z, w)$ is real valued; thus we can remove the complex conjugate in the integral above. For this and related results see Chapter 8 of [3]. From this it is easily checked that the harmonic Bergman projection $R: L^{2} \rightarrow b^{2}$ is the integral operator against the kernel $R(z, w)$ :

$$
R f(z)=\int_{H} f(w) R(z, w) d w
$$

for all $f \in L^{2}$. The explicit formula for the harmonic Bergman kernel is given by

$$
R(z, w)=\frac{4}{n \sigma_{n}} \frac{n\left(z_{n}+w_{n}\right)^{2}-|z-\bar{w}|^{2}}{|z-\bar{w}|^{n+2}} .
$$


Here, we use the notation $\bar{w}=\left(w^{\prime},-w_{n}\right)$ for $w \in H$. Note that if $n=2$, then $\bar{w}$ is the usual complex conjugate of $w$. From this formula we easily see that there is a constant $C=C(n)$ such that

$$
|R(z, w)| \leq \frac{C}{|z-\bar{w}|^{n}}
$$

for all $z, w \in H$. It is not hard to see that the function $w \mapsto|z-\bar{w}|^{-n q}$ is integrable for each $1<q<\infty$ and for each fixed $z$. Also note that $|R(z, w)| \leq C z_{n}^{-n}$ for all $w \in H$. Hence, inequality (2.4) shows that the harmonic Bergman projection $R$ extends to an integral operator, defined by (2.2), from $L^{p}$ into the space of harmonic functions on $H$ for each $1 \leq$ $p<\infty$. Moreover, $R: L^{p} \rightarrow b^{p}$ is a bounded projection for $1<p<\infty$, but for some $f \in L^{1}, R f$ is not even integrable. See [8] for more details.

In [8] it is shown that there are many other bounded projections from $L^{p}$ onto $b^{p}$. Let $D_{w_{n}}$ denote the differentiation with respect to the last component of $w$. In case there is no possibility of confusion, we will simply write $D$ for $D_{w_{n}}, D_{z_{n}}$, etc. One can easily check from (2.3) that

$$
D_{z_{n}} R(z, w)=D_{w_{n}} R(z, w) .
$$

Now, for each integer $k \geq 0$, define

$$
R_{k}(z, w)=\frac{(-2)^{k}}{k !} w_{n}^{k} D_{w_{n}}^{k} R(z, w)
$$

for $z, w \in H$. Note that $R_{0}(z, w)=R(z, w)$. This kernel $R_{k}(z, w)$ also has the following reproducing property as does $R(z, w)$ : If $1 \leq p<\infty$ and $u \in b^{p}$, then

$$
u(z)=\int_{H} u(w) R_{k}(z, w) d w
$$

for every $z \in H$. In fact, the kernel $R_{k}(z, w)$ has the following generalized reproducing property: If $1 \leq p<\infty$ and $u \in b^{p}$, then

$$
u(z)=\frac{(-2)^{m} k !}{(k+m) !} \int_{H} w_{n}^{m}\left[D^{m} u(w)\right] R_{k}(z, w) d w
$$

for every integer $m \geq 0$. Associated with the kernel $R_{k}(z, w)$ is the integral operator $R_{k}$ defined by the formula

$$
R_{k} f(z)=\int_{H} f(w) R_{k}(z, w) d w
$$


whenever the above integral makes sense. For $k \geq 1$, the kernel $R_{k}(z, w)$ behaves better than the harmonic Bergman kernel $R(z, w)$ in the sense that $R_{k}: L^{p} \rightarrow b^{p}$ is a bounded projection for every $1 \leq p<\infty$. For the proofs of these results, refer to [8]. In the same paper the Bergman norm is shown to be equivalent to the normal derivative norm: If $1 \leq p<\infty$ and $k \geq 0$ is an integer, then

$$
\|u\|_{p}^{p} \approx \int_{H} w_{n}^{p k}\left|D^{k} u(w)\right|^{p} d w
$$

as $u$ ranges over $b^{p}$. Here, and later, the notation $a(u) \approx b(u)$ means that the ratio $a(u) / b(u)$ is bounded above and below by some positive constants independent of $u$. The analogous result for the tangential derivative norm can also be found in [8].

We now summarize preliminary results on the harmonic Bloch space $\widetilde{\mathcal{B}}$. For a harmonic function $u$ on $H$, we say $u \in \mathcal{B}$ if

$$
\|u\|_{\mathcal{B}}=\sup _{w \in H} w_{n}|\nabla u(w)|<\infty
$$

Let $z_{0}=(0,1) \in H$. We will let $\widetilde{\mathcal{B}}$ denote the space of all functions $u \in \mathcal{B}$ such that $u\left(z_{0}\right)=0$. The space $\widetilde{\mathcal{B}}$ is a Banach space by a standard argument. We say that $u \in \widetilde{\mathcal{B}}_{0}$, the harmonic little Bloch space, if $u \in \widetilde{\mathcal{B}}$ satisfies the additional boundary vanishing condition

$$
\lim w_{n}|\nabla u(w)|=0
$$

where the limit is taken as $w \rightarrow \partial H \cup\{\infty\}$. It is not hard to verify that $\widetilde{\mathcal{B}}_{0}$ is a closed subspace of $\widetilde{\mathcal{B}}$. Although we do not need here, we remark in passing that $\widetilde{\mathcal{B}}$ is identified with the dual of $b^{1}$ in [8]. Also, $\widetilde{\mathcal{B}}_{0}$ is identified with the predual of $b^{1}$ in [10]. In proving these dualities, Ramey and Yi [8] introduced the modified kernel

$$
\widetilde{R}(z, w)=R(z, w)-R\left(z_{0}, w\right)
$$

to overcome the nonintegrability of $R(z, w)$. In fact, the kernel $\widetilde{R}(z, w)$ satisfies the estimate

$$
|\widetilde{R}(z, w)| \leq C \frac{\left|z-z_{0}\right|}{\left|z_{0}-\bar{w}\right|^{n+1}}\left(\frac{\left|z_{0}-\bar{w}\right|^{n}}{|z-\bar{w}|^{n}}+\frac{\left|z_{0}-\bar{w}\right|}{|z-\bar{w}|}\right)
$$

for some constant $C=C(n)$ and for all $z, w \in H$. Thus, the function $w \mapsto \widetilde{R}(z, w)$ is integrable over $H$ for each fixed $z$. This kernel $\widetilde{R}(z, w)$ 
turns out to be a reproducing kernel for the space $\widetilde{\mathcal{B}}$. More generally, for an integer $k \geq 0$, let

$$
\widetilde{R}_{k}(z, w)=R_{k}(z, w)-R_{k}\left(z_{0}, w\right)
$$

Then $\widetilde{R}_{k}(z, w)$ has the following reproducing property for harmonic Bloch functions: If $u \in \widetilde{\mathcal{B}}$, then

$$
u(z)=\int_{H} u(w) \widetilde{R}_{k}(z, w) d w
$$

for all $z \in H$. The generalized reproducing property of the kernel $\widetilde{R}_{k}(z, w)$ is also available:

$$
u(z)=\frac{(-2)^{m} k !}{(k+m) !} \int_{H} w_{n}^{m}\left[D^{m} u(w)\right] \widetilde{R}_{k}(z, w) d w
$$

for every integer $m \geq 0$ and for every $u \in \widetilde{\mathcal{B}}$. Also, the associated integral operator $\widetilde{R}_{k}$ defined by the formula

$$
\widetilde{R}_{k} f(z)=\int_{H} f(w) \widetilde{R}_{k}(z, w) d w
$$

takes $L^{\infty}$ onto $\widetilde{\mathcal{B}}$ boundedly. These results were proved in [8] only for the case $k=0$. In order to prove the remaining cases, one can modify the ideas of [8] which are used to prove the $L^{p}$-boundedness of $R_{k}$ and the generalized reproducing property $(2.6)$.

The Bloch norm is also equivalent to the normal derivative norm ([8]): If $k \geq 1$ is an integer, then

$$
\|u\|_{\mathcal{B}} \approx \sup _{w \in H} w_{n}^{k}\left|D^{k} u(w)\right|
$$

as $u$ ranges over $\widetilde{\mathcal{B}}$. Also, for $u \in \widetilde{\mathcal{B}}$, we have $u \in \widetilde{\mathcal{B}_{0}}$ if and only if

$$
\lim w_{n}^{k} D^{k} u(w)=0
$$

where the limit is taken as $w \rightarrow \partial H \cup\{\infty\}$. See [10]. 


\section{$\S 3$. Basic Lemmas}

Words on Constants. In the rest of the paper the same letter $C$ will denote various positive constants, unless otherwise specified, which may change at each occurrence. The constant $C$ may often depend on the dimension $n$ and some other parameters like $\delta, p, k$, or $\alpha$, but it will be always independent of particular functions, points or sequences under consideration.

In this section we prove several basic lemmas which will be used in later sections. We first introduce a distance function on $H$ which is useful for our purposes. In the hyperbolic geometry of $H$, the arclength element is $|d \vec{x}| / x_{n}$ and geodesics are (i) vertical lines and (ii) semi-circles centered on and orthogonal to $\mathbb{R}^{n-1}$. Thus, one can verify that the hyperbolic distance between two points $z, w \in H$ is

$$
\log \frac{1+\rho(z, w)}{1-\rho(z, w)}
$$

where

$$
\rho(z, w)=\frac{|z-w|}{|z-\bar{w}|} .
$$

We shall work with this pseudohyperbolic distance $\rho$. While the pseudohyperbolic distance is well known on the upper half-plane (see, for example, $[6]$ ), it appears to be not widely known in general. Here, we include a direct proof that $\rho$ indeed defines a distance function on $H$. First, note that $\rho$ is horizontal translation invariant and dilation invariant. In particular,

$$
\rho(z, w)=\rho\left(\phi_{a}(z), \phi_{a}(w)\right) \quad(z, w \in H)
$$

where $\phi_{a}(a \in H)$ denotes the function defined by

$$
\phi_{a}(z)=\left(\frac{z^{\prime}-a^{\prime}}{a_{n}}, \frac{z_{n}}{a_{n}}\right)
$$

for $z=\left(z^{\prime}, z_{n}\right) \in H$. Recall $z_{0}=(0,1)$.

LEMma 3.1. $\rho$ is a distance function on $H$.

Proof. Of course, we only need to check the triangle inequality. From (3.1), we only need to show

$$
\rho\left(z, z_{0}\right) \leq \rho(z, w)+\rho\left(w, z_{0}\right)
$$


for $z, w \in H$. Fix $z, w \in H$. Let $\rho_{2}$ denote the pseudohyperbolic distance on the upper half-plane. Then we get

$$
\begin{aligned}
\rho\left(z, z_{0}\right) & =\rho_{2}\left(\left(\left|z^{\prime}\right|, z_{n}\right),(0,1)\right) \\
& \leq \rho_{2}\left(\left(\left|z^{\prime}\right|, z_{n}\right),\left(\left|w^{\prime}\right|, w_{n}\right)\right)+\rho_{2}\left(\left(\left|w^{\prime}\right|, w_{n}\right),(0,1)\right) \\
& =\rho_{2}\left(\left(\left|z^{\prime}\right|, z_{n}\right),\left(\left|w^{\prime}\right|, w_{n}\right)\right)+\rho\left(w, z_{0}\right) .
\end{aligned}
$$

Note that

$$
\begin{aligned}
\rho_{2}\left(\left(\left|z^{\prime}\right|, z_{n}\right),\left(\left|w^{\prime}\right|, w_{n}\right)\right) & =\rho_{2}\left(\left(\frac{\left|z^{\prime}\right|-\left|w^{\prime}\right|}{w_{n}}, \frac{z_{n}}{w_{n}}\right),(0,1)\right) \\
& \leq \rho_{2}\left(\left(\frac{\left|z^{\prime}-w^{\prime}\right|}{w_{n}}, \frac{z_{n}}{w_{n}}\right),(0,1)\right) \\
& =\rho(z, w)
\end{aligned}
$$

so that (3.3) holds. The proof is complete.

For $z \in H$ and $0<\delta<1$, let $E_{\delta}(z)$ denote the pseudohyperbolic ball centered at $z$ with radius $\delta$. Note that $\phi_{z}\left(E_{\delta}(z)\right)=E_{\delta}\left(z_{0}\right)$ by the invariance property (3.1). Also, a straightforward calculation shows that

$$
E_{\delta}(z)=B\left(\left(z^{\prime}, \frac{1+\delta^{2}}{1-\delta^{2}} z_{n}\right), \frac{2 \delta}{1-\delta^{2}} z_{n}\right)
$$

so that $B\left(z, \delta z_{n}\right) \subset E_{\delta}(z) \subset B\left(z, 2 \delta(1-\delta)^{-1} z_{n}\right)$ where $B(z, r)$ denotes the euclidean ball centered at $z$ with radius $r$. We first prove two simple consequences of (3.4).

Lemma 3.2. For $z, w \in H$, we have

$$
\frac{1-\rho(z, w)}{1+\rho(z, w)} \leq \frac{z_{n}}{w_{n}} \leq \frac{1+\rho(z, w)}{1-\rho(z, w)}
$$

Proof. Assume $\rho(z, w)<\delta$. Then, by (3.4),

$$
\frac{z_{n}}{w_{n}}<\frac{1+\delta}{1-\delta}
$$

This implies the lemma.

Lemma 3.3. For $z, w \in H$, we have

$$
\frac{1-\rho(z, w)}{1+\rho(z, w)} \leq \frac{|z-\bar{s}|}{|w-\bar{s}|} \leq \frac{1+\rho(z, w)}{1-\rho(z, w)}
$$

for all $s \in H$. 
Proof. Assume $\rho(z, w)<\delta$ and let $s \in H$. Then

$$
|z-\bar{s}|-|w-\bar{s}| \leq|w-z|
$$

On the other hand, since $E_{\delta}(w) \subset B\left(w, 2 \delta(1-\delta)^{-1} w_{n}\right)$, we have

$$
|w-z| \leq \frac{2 \delta}{1-\delta} w_{n} \leq \frac{2 \delta}{1-\delta}|w-\bar{s}|
$$

Insert this into (3.5) and get

$$
\frac{|z-\bar{s}|}{|w-\bar{s}|} \leq \frac{1+\delta}{1-\delta}
$$

This proves the lemma.

Note that the kernel $R(z, w)$ has zeros on $H \times H$. Thus, some estimates on holomorphic Bergman kernels in [5], [9] have no harmonic analogue. Instead we have the following estimate.

LEMMA 3.4. Let $k \geq 0$ be an integer and $\alpha$ be real. Then

$$
\left|z_{n}^{\alpha} R_{k}(s, z)-w_{n}^{\alpha} R_{k}(s, w)\right| \leq C \rho(z, w) \frac{z_{n}^{k+\alpha}}{|z-\bar{s}|^{n+k}}
$$

whenever $\rho(z, w)<1 / 2$ and $s \in H$.

Proof. Assume $\rho(z, w)<1 / 2$ and let $s \in H$. We first show that

$$
\left|R_{k}(s, z)-R_{k}(s, w)\right| \leq C \rho(z, w) \frac{z_{n}^{k}}{|z-\bar{s}|^{n+k}} .
$$

By induction one can check that $R_{k}(s, z)$ can be written in the form

$$
R_{k}(s, z)=z_{n}^{k} \sum_{m=0}^{k+2} c_{m} \frac{\left(s_{n}+z_{n}\right)^{m}}{|z-\bar{s}|^{n+k+m}}
$$

for some coefficients $c_{m}$. Note that this implies

$$
\left|R_{k}(s, z)\right| \leq C \frac{z_{n}^{k}}{|z-\bar{s}|^{n+k}}
$$


Now, to prove (3.6), it is sufficient to show

$$
\left|\frac{s_{n}^{i} z_{n}^{j+k}}{|z-\bar{s}|^{n+k+m}}-\frac{s_{n}^{i} w_{n}^{j+k}}{|w-\bar{s}|^{n+k+m}}\right| \leq C \rho(z, w) \frac{z_{n}^{k}}{|z-\bar{s}|^{n+k}}
$$

for $0 \leq i+j=m \leq k+2$. The left side of the above is less than or equal to

$$
\begin{aligned}
& \frac{\left|s_{n}^{i} z_{n}^{j+k}-s_{n}^{i} w_{n}^{j+k}\right|}{|z-\bar{s}|^{n+k+m}}+\left|\frac{s_{n}^{i} w_{n}^{j+k}}{|z-\bar{s}|^{n+k+m}}-\frac{s_{n}^{i} w_{n}^{j+k}}{|w-\bar{s}|^{n+k+m}}\right| \\
& \quad \leq \frac{s_{n}^{i} z_{n}^{j+k}}{|z-\bar{s}|^{n+k+m}}\left|1-\left(\frac{w_{n}}{z_{n}}\right)^{j+k}\right|+\frac{s_{n}^{i} w_{n}^{j+k}}{|z-\bar{s}|^{n+k+m}}\left|1-\left(\frac{|z-\bar{s}|}{|w-\bar{s}|}\right)^{n+k+m}\right| \\
& \quad \leq \frac{z_{n}^{k}}{|z-\bar{s}|^{n+k}}\left\{\left|1-\left(\frac{w_{n}}{z_{n}}\right)^{j+k}\right|+\left(\frac{w_{n}}{z_{n}}\right)^{k}\left|1-\left(\frac{|z-\bar{s}|}{|w-\bar{s}|}\right)^{n+k+m}\right|\right\} .
\end{aligned}
$$

Since $\rho(z, w)<1 / 2$, by Lemma 3.2 , we have $1 / 3<w_{n} / z_{n}<3$ and hence the first term inside the parenthesis of the above is dominated by some constant independent of $z, w$. Similarly, it follows from Lemma 3.3 that the second term inside of the parenthesis of the above is dominated by some constant independent of $z, w$. This proves (3.8) and thus (3.6).

Now, let $\alpha$ be a given real number. By (3.6) and (3.7), we have

$$
\begin{aligned}
& \left|z_{n}^{\alpha} R_{k}(s, z)-w_{n}^{\alpha} R_{k}(s, w)\right| \\
& \quad \leq z_{n}^{\alpha}\left|R_{k}(s, z)-R_{k}(s, w)\right|+z_{n}^{\alpha}\left|R_{k}(s, w)\right|\left|1-\left(\frac{w_{n}}{z_{n}}\right)^{\alpha}\right| \\
& \quad \leq C \frac{z_{n}^{k+\alpha}}{|z-\bar{s}|^{n+k}}+C \frac{z_{n}^{k+\alpha}}{|w-\bar{s}|^{n+k}} \\
& \quad \leq C \frac{z_{n}^{k+\alpha}}{|z-\bar{s}|^{n+k}} .
\end{aligned}
$$

The last two inequalities of the above hold by Lemma 3.2 and Lemma 3.3. The proof is complete.

Estimate like (2.1) and Cauchy's estimates yield the following lemma whose proof can be found in Corollary 8.2 of [3]. The notation $d(E, F)$ denotes the euclidean distance between two sets $E$ and $F$.

LEMMA 3.5. Suppose $u$ is harmonic on some proper open subset $\Omega$ of $\mathbb{R}^{n}$. Let $1 \leq p<\infty$ and $\alpha=\left(\alpha_{1}, \cdots, \alpha_{n}\right)$ be a multi-index of nonnegative 
integers. Then

$$
\left|\frac{\partial^{|\alpha|} u}{\partial x_{1}^{\alpha_{1}} \cdots \partial x_{n}^{\alpha_{n}}}(a)\right|^{p} \leq \frac{C}{d(a, \partial \Omega)^{n+p|\alpha|}} \int_{\Omega}|u|^{p} d V
$$

for all $a \in \Omega$. The constant $C$ depends only on $n, p$ and $\alpha$.

Taking $\Omega=E_{\delta}(a)$ in the above lemma, we have the growth estimate for normal derivatives of harmonic functions on $H$.

LEMMA 3.6. Let $1 \leq p<\infty, 0<\delta<1$, and $k \geq 0$ be an integer. Then

$$
a_{n}^{n+p k}\left|D^{k} u(a)\right|^{p} \leq \frac{C}{\delta^{n+p k}} \int_{E_{\delta}(a)}|u(w)|^{p} d w
$$

for all $a \in H$ and for every $u$ harmonic on $H$. The constant $C=C(n, k, p)$ is independent of $\delta$.

Proof. Let $u$ be a harmonic function on $H$ and fix $a \in H$. From (3.4), it is easily seen $d\left(z_{0}, \partial E_{\delta}\left(z_{0}\right)\right)=2 \delta /(1+\delta)$. Thus, by Lemma 3.5 , we have

$$
\left|D^{k} u\left(z_{0}\right)\right|^{p} \leq \frac{C}{\delta^{n+p k}} \int_{E_{\delta}\left(z_{0}\right)}|u(w)|^{p} d w
$$

for some constant $C=C(n, p, k)$. Given $a \in H$, apply the above inequality to $u \circ \phi_{a}^{-1}$ where $\phi_{a}$ is the map defined in (3.2) and then make a change of variables $z=\phi_{a}^{-1}(w)$. The result is

$$
a_{n}^{p k}\left|D^{k} u(a)\right|^{p} \leq \frac{C}{a_{n}^{n} \delta^{n+p k}} \int_{E_{\delta}(a)}|u(z)|^{p} d z
$$

which completes the proof.

By Lemma 3.5, derivatives of harmonic functions cannot grow arbitrarily, and neither can integrals of their oscillations. In what follows $|K|$ denotes the Lebesgue measure of a Borel subset $K$ of $H$.

LEMMA 3.7. Suppose $u$ is harmonic on some proper open subset $\Omega$ of $\mathbb{R}^{n}$ and let $1 \leq p<\infty$. Then, for a given open ball $E \subset \Omega$,

$$
\int_{E}|u(z)-u(a)|^{p} d z \leq C \frac{|E|^{1+p / n}}{d(E, \partial \Omega)^{n+p}} \int_{\Omega}|u|^{p} d V
$$

for all $a \in E$. The constant $C$ depends only on $n$ and $p$. 
Proof. Fix $a \in E$. Then, we have

$$
|u(z)-u(a)| \leq|z-a| \sup _{w \in E}|\nabla u(w)|
$$

for all $z \in E$. Note that, for $w \in E$,

$$
\begin{aligned}
|\nabla u(w)| & \leq \frac{C}{d(w, \partial \Omega)^{1+n / p}}\left(\int_{\Omega}|u|^{p} d V\right)^{1 / p} \\
& \leq \frac{C}{d(E, \partial \Omega)^{1+n / p}}\left(\int_{\Omega}|u|^{p} d V\right)^{1 / p}
\end{aligned}
$$

by Lemma 3.5. Hence,

$$
|u(z)-u(a)|^{p} \leq C \frac{|z-a|^{p}}{d(E, \partial \Omega)^{n+p}} \int_{\Omega}|u|^{p} d V .
$$

Since $|z-a|^{n} \leq C|E|$ for every $a, z \in E$, we conclude from (3.9)

$$
\begin{aligned}
\int_{E}|u(z)-u(a)|^{p} d z & \leq \frac{C}{d(E, \partial \Omega)^{n+p}} \int_{E}|z-a|^{p} d z \int_{\Omega}|u|^{p} d V \\
& \leq C \frac{|E|^{1+p / n}}{d(E, \partial \Omega)^{n+p}} \int_{\Omega}|u|^{p} d V
\end{aligned}
$$

as desired. The proof is complete.

Remark 1. By the fact that $|u|^{p}$ is almost subharmonic (see Lemma 3.5 of [5]) for $0<p<1$, Lemma 3.5, Lemma 3.6, and Lemma 3.7 are actually true for all $0<p<\infty$. However, we will not need this fact in the present paper.

We now close this section with two simple integral formulas which are useful in understanding the integral behaviour of various kernels.

Lemma 3.8. For $\alpha>0$, we have

$$
\int_{H} \frac{d w}{|z-\bar{w}|^{n+\alpha}}=C z_{n}^{-\alpha}
$$

for every $z \in H$. 
Proof. Fix $z$ and substitute $w=\phi_{z}^{-1}(s)=\left(z_{n} s^{\prime}+z^{\prime}, z_{n} s_{n}\right)$ to see that

$$
\int_{H} \frac{d w}{|z-\bar{w}|^{n+\alpha}}=z_{n}^{-\alpha} \int_{H} \frac{d s}{\left|s-\bar{z}_{0}\right|^{n+\alpha}} .
$$

Now, the lemma follows from the estimate

$$
\int_{H} \frac{d s}{\left|s-\bar{z}_{0}\right|^{n+\alpha}} \leq \int_{H} \frac{d s}{\left[1+|s|^{2}\right]^{(n+\alpha) / 2}}=C \int_{0}^{\infty} \frac{r^{n-1}}{\left[1+r^{2}\right]^{(n+\alpha) / 2}} d r<\infty .
$$

The proof is complete.

For a proof of the following lemma, see Lemma 3.1 of [8].

LEMMA 3.9. For $0<\alpha<1$, we have

$$
\int_{H} \frac{w_{n}^{-\alpha}}{|z-\bar{w}|^{n}} d w=C z_{n}^{-\alpha}
$$

for every $z \in H$.

\section{§4. Representations}

For a motivation, consider a sequence $\left\{z_{m}\right\}$ of distinct points in $H$ with $z_{m} \rightarrow \partial H \cup\{\infty\}$ and pick a pairwise disjoint covering $\left\{E_{m}\right\}$ of $H$ such that $z_{m} \in E_{m}$. For an integer $k \geq 0$ and $u \in b^{p}$, we see from the reproducing property (2.5)

$$
u(z)=\sum \int_{E_{m}} u(w) R_{k}(z, w) d w .
$$

Let $q$ be the conjugate exponent of $p$. Then, the series

$$
\sum u\left(z_{m}\right)\left|E_{m}\right|^{1 / p} \cdot\left|E_{m}\right|^{1 / q} R_{k}\left(z, z_{m}\right)
$$

can be considered as an approximating Riemann sum of the above integral. Note that the sum

$$
\sum\left|u\left(z_{m}\right)\right|^{p}\left|E_{m}\right|
$$

can be viewed as an approximating Riemann sum of $\|u\|_{p}^{p}$. In this section we find a sufficient condition on the sequence $\left\{z_{m}\right\}$ for which every function $u \in b^{p}$ can be represented in this way. By intuition, points of such a sequence are expected to be sufficiently dense so that approximating Riemann sums get very close to actual integrals. This intuition has been already made precise for holomorphic Bergman functions. See [5], [7]. We will prove 
that "sufficient density" remains still sufficient for representation on the setting of the present paper. Also, we consider an analogous representation property on the limiting spaces $\widetilde{\mathcal{B}}$ and $\widetilde{\mathcal{B}}_{0}$.

In the holomorphic case $([5],[7])$, representation theorems were proved under the lattice density condition. Representation theorems for harmonic Bergman functions on the setting of the unit ball are also proved in [5] under the similar lattice density condition. The analogous lattice density condition will turn out to be sufficient for representation of $b^{p}$-functions. The argument of [5] is a constructive one, while that of [7] extensively uses dualities. We will take the more constructive idea of [5]. To be more precise, let us introduce some terminology. Let $\left\{z_{m}\right\}$ be a sequence in $H$ and $0<\delta<1$. We say that $\left\{z_{m}\right\}$ is $\delta$-separated if the balls $E_{\delta}\left(z_{m}\right)$ are pairwise disjoint or simply say that $\left\{z_{m}\right\}$ is separated if it is $\delta$-separated for some $\delta$. Also, we say that $\left\{z_{m}\right\}$ is a $\delta$-lattice if it is $\delta / 2$-separated and $H=\cup E_{\delta}\left(z_{m}\right)$. Note that any "maximal" $\delta / 2$-separated sequence is a $\delta$ lattice. Also, one can explicitly construct a $\delta$-lattice by using almost the same argument of [5]. We will prove that $\delta$-lattices, with $\delta$ sufficiently small, are representing sequences. The main tool in proving such representation results is the following covering lemma. One can also prove it by using almost the same argument of [5].

Lemma 4.1. Fix a $1 / 2$-lattice $\left\{a_{m}\right\}$ and let $0<\delta<1 / 8$. If $\left\{z_{m}\right\}$ is a $\delta$ lattice, then we can find a rearrangement $\left\{z_{i j} \mid i=1,2, \ldots, j=1,2, \ldots, N_{i}\right\}$ of $\left\{z_{m}\right\}$ and a pairwise disjoint covering $\left\{D_{i j}\right\}$ of $H$ with the following properties:

$$
\begin{aligned}
& E_{\delta / 2}\left(z_{i j}\right) \subset D_{i j} \subset E_{\delta}\left(z_{i j}\right) \\
& E_{1 / 4}\left(a_{i}\right) \subset \cup_{j=1}^{N_{i}} D_{i j} \subset E_{5 / 8}\left(a_{i}\right) \\
& z_{i j} \in E_{1 / 2}\left(a_{i}\right)
\end{aligned}
$$

for all $i=1,2, \cdots$, and $j=1,2, \ldots, N_{i}$.

By property (c) of the above, the sequence $N_{i}$ must have an upper bound, because pseudohyperbolic balls centered at lattice points cannot intersect too often as the following lemma shows.

LEMmA 4.2. Let $\alpha>0$ and assume $0<(1+\alpha) \eta<1$. If $\left\{z_{m}\right\}$ is an $\eta$-separated sequence, then there is a constant $M=M(n, \alpha, \eta)$ such that more than $M$ of the balls $E_{\alpha \eta}\left(z_{m}\right)$ contain no point in common. 
Proof. The proof is a standard volume argument. So, first note from (3.4) that there exists a positive constant $C=C(n)$ such that

$$
C^{-1} r^{n} \leq a_{n}^{-n}\left|E_{r}(a)\right| \leq C\left(\frac{r}{1-r}\right)^{n}
$$

for all $0<r<1$ and $a \in H$. Fix $z$ and let $J_{z}$ be set of all indices $m$ such that $\rho\left(z_{m}, z\right)<\alpha \eta$. Note that $E_{\eta}\left(z_{m}\right) \subset E_{(1+\alpha) \eta}(z)$ for each $m \in J_{z}$. Therefore, by the disjointness of the balls $E_{\eta}\left(z_{m}\right)$, we have

$$
\sum_{m \in J_{z}}\left|E_{\eta}\left(z_{m}\right)\right| \leq\left|E_{(1+\alpha) \eta}(z)\right|
$$

Also, for each $m \in J_{z}$, we have by Lemma 3.2

$$
\frac{1-\alpha \eta}{1+\alpha \eta} \leq \frac{z_{m n}}{z_{n}}
$$

Letting $M_{z}$ denote the cardinality of $J_{z}$, we obtain from (4.2), (4.3), (4.4) that

$$
M_{z} \eta^{n} z_{n}^{n}\left(\frac{1-\alpha \eta}{1+\alpha \eta}\right)^{n} \leq C \sum_{m \in J_{z}} \eta^{n} z_{m n}^{n} \leq C\left(\frac{(1+\alpha) \eta}{1-(1+\alpha) \eta}\right)^{n} z_{n}^{n}
$$

so that

$$
M_{z} \leq C\left(\frac{1+\alpha}{1-(1+\alpha) \eta}\right)^{n}\left(\frac{1+\alpha \eta}{1-\alpha \eta}\right)^{n}
$$

for some constant $C$ depending only on $n$. The proof is complete.

LEMMA 4.3. Let $N_{i}$ be the sequence defined in Lemma 4.1. Then

$$
\sup _{i} N_{i} \leq C \delta^{-n}
$$

for some constant $C$ depending only on $n$.

Proof. By (c), each $a_{i}$ is contained in $E_{1 / 2}\left(z_{i j}\right)$ for each $j=1,2, \cdots, N_{i}$. Hence, by Lemma 4.2 with $\eta=\delta / 2$ and $\alpha=1 / \delta$, we obtain from (4.5) (recall $0<\delta<1 / 8)$

$$
N_{i} \leq C\left(1+\delta^{-1}\right)^{n}(1-\delta)^{-n} \leq C \delta^{-n}
$$

for some constant $C$ depending only on $n$. 
Representation on $b^{p}$

Let $\left\{z_{m}\right\}$ be a sequence in $H$. Let $1 \leq p<\infty$ and $k \geq 0$ be an integer. For $\left(\lambda_{m}\right) \in l^{p}$, let $Q_{k}\left(\lambda_{m}\right)$ denote the series defined by

$$
Q_{k}\left(\lambda_{m}\right)(z)=\sum \lambda_{m} z_{m n}^{n(1-1 / p)} R_{k}\left(z, z_{m}\right) \quad(z \in H)
$$

Here, we restrict $k \geq 1$ for $p=1$. For a sequence $\left\{z_{m}\right\}$ good enough, $Q_{k}\left(\lambda_{m}\right)$ will be harmonic on $H$. We say that $\left\{z_{m}\right\}$ is a $b^{p}$-representing sequence of order $k$ if $Q_{k}\left(l^{p}\right)=b^{p}$.

Of course, the motivation for the series (4.6) is the approximating Riemann sum (4.1) where $E_{m}$ is pretended to be the ball $E_{\delta}\left(z_{m}\right)$ for some fixed $\delta$. However, it might not be clear from the very definition that the series (4.6) defines a $b^{p}$-function under the separation condition. To make this clear, we need a lemma which is proved in the course of the proof of Theorem 3.2 and Theorem 4.2 of [8]. We prove it here for the reader's convenience.

Lemma 4.4. Let $1 \leq p<\infty$ and $k \geq 0$ be an integer. For $f \in L^{p}$, define

$$
\Phi_{k} f(z)=\int_{H} f(w) \frac{w_{n}^{k}}{|z-\bar{w}|^{n+k}} d w \quad(z \in H) .
$$

Then, for $1<p<\infty, \Phi_{k}: L^{p} \rightarrow L^{p}$ is bounded for each $k \geq 0$. Also, $\Phi_{k}: L^{1} \rightarrow L^{1}$ is bounded for each $k \geq 1$.

Proof. First assume $p>1$ and $k \geq 0$. Since $\left|\Phi_{k} f\right| \leq\left|\Phi_{0}\right| f \|$, it is enough to show $\Phi_{0}: L^{p} \rightarrow L^{p}$ is bounded. Let $q$ be the conjugate exponent of $p$. Note that

$$
\frac{|f(w)|}{|z-\bar{w}|^{n}}=|f(w)| \frac{w_{n}^{1 / p q}}{|z-\bar{w}|^{n / p}} \cdot \frac{w_{n}^{-1 / p q}}{|z-\bar{w}|^{n / q}} .
$$

Thus, taking absolute values, and then applying Hölder's inequality and Lemma 3.9, we obtain

$$
\begin{aligned}
\left|\Phi_{0} f(z)\right|^{p} & \leq\left\{\int_{H}|f(w)|^{p} \frac{w_{n}^{1 / q}}{|z-\bar{w}|^{n}} d w\right\}\left\{\int_{H} \frac{w_{n}^{-1 / p}}{|z-\bar{w}|^{n}} d w\right\}^{p / q} \\
& \leq C z_{n}^{-1 / q} \int_{H}|f(w)|^{p} \frac{w_{n}^{1 / q}}{|z-\bar{w}|^{n}} d w .
\end{aligned}
$$


Integrate both sides of the above. After interchanging the order of integration, we see from Lemma 3.9 once more

$$
\begin{aligned}
\int_{H}\left|\Phi_{0} f(z)\right|^{p} d z & \leq C \int_{H}|f(w)|^{p} w_{n}^{1 / q} \int_{H} \frac{z_{n}^{-1 / q}}{|z-\bar{w}|^{n}} d z d w \\
& \leq C \int_{H}|f(w)|^{p} d w
\end{aligned}
$$

Next, for $p=1$ and $k \geq 1$, it is enough to show $\Phi_{1}: L^{1} \rightarrow L^{1}$ is bounded. By using Fubini's theorem and Lemma 3.8, we have

$$
\begin{aligned}
\int_{H}\left|\Phi_{1} f(z)\right| d z & \leq \int_{H} \int_{H}|f(w)| \frac{w_{n}}{|z-\bar{w}|^{n+1}} d w d z \\
& =\int_{H}|f(w)| \int_{H} \frac{w_{n}}{|z-\bar{w}|^{n+1}} d z d w \\
& \leq C \int_{H}|f(w)| d w
\end{aligned}
$$

The proof is complete.

The following shows $Q_{k}\left(l^{p}\right) \subset b^{p}$ if the underlying sequence is separated. In other words, every separated sequence represents a part of the whole space.

Proposition 4.5. Let $1 \leq p<\infty$ and $k \geq 0$ be an integer. Suppose $\left\{z_{m}\right\}$ is a $\delta$-separated sequence. Let $Q_{k}$ be the associated operator as in (4.6). Then, for $1<p<\infty, Q_{k}: l^{p} \rightarrow b^{p}$ is bounded for each $k \geq 0$. Also, $Q_{k}: l^{1} \rightarrow b^{1}$ is bounded for each $k \geq 1$.

Proof. By (3.7), Lemma 3.2 and Lemma 3.3, there exists a constant $C=C(n, k, \delta)$ such that

$$
\left|R_{k}\left(z, z_{m}\right)\right| \leq C \frac{z_{m n}^{k}}{\left|z-\bar{z}_{m}\right|^{n+k}} \leq C \frac{s_{n}^{k}}{|z-\bar{s}|^{n+k}}
$$

for all $s \in E_{\delta}\left(z_{m}\right)$ and $z \in H$. Let $\chi_{m}$ denote the characteristic function of $E_{\delta}\left(z_{m}\right)$ and, for $\left(\lambda_{m}\right) \in l^{p}$, put

$$
f=\sum\left|\lambda_{m}\right| z_{m n}^{n(1-1 / p)}\left|E_{\delta}\left(z_{m}\right)\right|^{-1} \chi_{m}
$$


Then, by (4.7), we have

$$
\begin{aligned}
\left|Q_{k}\left(\lambda_{m}\right)(z)\right| & \leq C \sum\left|\lambda_{m}\right| z_{m n}^{n(1-1 / p)}\left|E_{\delta}\left(z_{m}\right)\right|^{-1} \int_{E_{\delta}\left(z_{m}\right)} \frac{s_{n}^{k}}{|z-\bar{s}|^{n+k}} d s \\
& =C \Phi_{k} f(z) .
\end{aligned}
$$

Now assume $k \geq 0$ for $1<p<\infty$ and assume $k \geq 1$ for $p=1$. Then, from Lemma 4.4, it follows that

$$
\begin{aligned}
\left\|Q_{k}\left(\lambda_{m}\right)\right\|_{p}^{p} & \leq C\|f\|_{p}^{p} \\
& =C \sum\left|\lambda_{m}\right|^{p} z_{m n}^{n(p-1)}\left|E_{\delta}\left(z_{m}\right)\right|^{1-p} \\
& \leq C \sum\left|\lambda_{m}\right|^{p}
\end{aligned}
$$

which shows that $Q_{k}: l^{p} \rightarrow L^{p}$ is bounded and the series in (4.6) converges in norm. Since every term in the series (4.6) is harmonic, the series converges uniformly on compact subsets of $H$. It follows that $Q_{k}$ maps $l^{p}$ into $b^{p}$. The proof is complete.

We are now ready to prove our $b^{p}$-representation theorem under the lattice density condition. Roughly speaking, it states that every sufficiently dense lattice represents the whole space. We first consider the case $1<p<$ $\infty$.

THEOREM 4.6. Let $1<p<\infty$ and let $k \geq 0$ be an integer. Then there exists a positive number $\delta_{0}$ with the following property: Let $\left\{z_{m}\right\}$ be a $\delta$-lattice with $\delta<\delta_{0}$ and let $Q_{k}: l^{p} \rightarrow b^{p}$ be the associated linear operator as in (4.6). Then there is a bounded linear operator $P_{k}: b^{p} \rightarrow l^{p}$ such that $Q_{k} P_{k}$ is the identity on $b^{p}$. In particular, $\left\{z_{m}\right\}$ is a $b^{p}$-representing sequence of order $k$.

This should be compared with Theorem 3 of Coifman and Rochberg [5]. While their theorem (on the ball) has the advantage of being valid for $p<1$, it contains the restriction $k \geq 1$ for $1<p<\infty$.

Proof. We may assume $\delta<1 / 8$. Fix a $1 / 2$-lattice $\left\{a_{m}\right\}$. Find a rearrangement $\left\{z_{i j}\right\}$ of $\left\{z_{m}\right\}$, as well as a pairwise disjoint covering $\left\{D_{i j}\right\}$ of $H$, for which all properties of Lemma 4.1 are satisfied. 
For $u \in b^{p}$, let $T u$ denote the sequence whose components are $u\left(z_{i j}\right)$ $\left|D_{i j}\right| z_{i j}^{-n / q}$ where $q$ is the conjugate exponent of $p$. By (a) and Lemma 3.6 we have

$$
\left|u\left(z_{i j}\right)\right|^{p} \leq \frac{C}{\left|D_{i j}\right|} \int_{D_{i j}}|u(w)|^{p} d w
$$

and therefore

$$
\sum\left|D_{i j}\right|^{p} z_{i j}^{n(1-p)}\left|u\left(z_{i j}\right)\right|^{p} \leq C \sum \int_{D_{i j}}|u|^{p} d V=C \int_{H}|u|^{p} d V
$$

which means the operator $T: b^{p} \rightarrow l^{p}$ is bounded and thus $Q_{k} T$ is bounded on $b^{p}$ by Proposition 4.5.

Now, we show that $Q_{k} T$ is invertible on $b^{p}$ for all $\delta$ sufficiently small. Let $\chi_{i j}$ denote the characteristic function of $D_{i j}$. The reproducing property (2.5) then gives

$$
u=R_{k} u=R_{k}\left[\sum u \chi_{i j}\right]=\sum R_{k}\left[u \chi_{i j}\right]
$$

Notice that

$$
Q_{k} T u(z)=\sum u\left(z_{i j}\right) R_{k}\left(z, z_{i j}\right)\left|D_{i j}\right| \text {. }
$$

Thus, we have $u-Q_{k} T u=u_{1}+u_{2}$ where

$$
\begin{aligned}
& u_{1}(z)=R_{k}\left[\sum\left(u-u\left(z_{i j}\right)\right) \chi_{i j}\right](z) \\
& u_{2}(z)=\sum u\left(z_{i j}\right) \int_{D_{i j}} R_{k}(z, w)-R_{k}\left(z, z_{i j}\right) d w
\end{aligned}
$$

To estimate these functions, first note that

$$
1 / 5<\frac{s_{n}}{a_{\text {in }}}<5, \quad 1 / 5<\frac{|z-\bar{s}|}{\left|z-\bar{a}_{i}\right|}<5
$$

for all $s \in E_{2 / 3}\left(a_{i}\right)$ and $z \in H$ by Lemma 3.2 and Lemma 3.3. Put $E_{i j}=$ $E_{\delta}\left(z_{i j}\right)$ and $E_{i}^{*}=E_{2 / 3}\left(a_{i}\right)$ for simplicity. Note $E_{i j} \subset E_{1 / 2+\delta}\left(a_{i}\right) \subset E_{5 / 8}\left(a_{i}\right)$ by (c). Hence, by (4.9),

$$
d\left(E_{i j}, \partial E_{i}^{*}\right) \geq d\left(E_{5 / 8}\left(a_{i}\right), \partial E_{i}^{*}\right) \geq C a_{i n} \geq C z_{i j n}
$$


for some absolute constant $C$. Thus, by (a) and Lemma 3.7, we find

$$
\begin{aligned}
\int_{D_{i j}}\left|u(w)-u\left(z_{i j}\right)\right|^{p} d w & \leq \int_{E_{i j}}\left|u(w)-u\left(z_{i j}\right)\right|^{p} d w \\
& \leq C \frac{\left|E_{i j}\right|^{1+p / n}}{d\left(E_{i j}, \partial E_{i}^{*}\right)^{n+p}} \int_{E_{i}^{*}}|u(w)|^{p} d w \\
& \leq C \delta^{n+p} \int_{E_{i}^{*}}|u(w)|^{p} d w
\end{aligned}
$$

for all $i, j$. Here, the constant $C$ is independent of $i, j, \delta$. Thus, for each fixed $i$, we obtain from Lemma 4.3

$$
\sum_{j=1}^{N_{i}} \int_{D_{i j}}\left|u(w)-u\left(z_{i j}\right)\right|^{p} d w \leq C \delta^{p} \int_{E_{i}^{*}}|u(w)|^{p} d w
$$

Now, since $R_{k}$ is $L^{p}$-bounded, we have

$$
\begin{aligned}
\left\|u_{1}\right\|_{p}^{p} & \leq C\left\|\sum_{i, j}\left(u-u\left(z_{i j}\right)\right) \chi_{i j}\right\|_{p}^{p} \\
& =C \sum_{i, j} \int_{D_{i j}}\left|u(w)-u\left(z_{i j}\right)\right|^{p} d w \\
& \leq C \delta^{p} \sum_{i} \int_{E_{i}^{*}}|u(w)|^{p} d w \\
& \leq C \delta^{p} \int_{H}|u(w)|^{p} d w .
\end{aligned}
$$

The last inequality of the above holds by Lemma 4.2. In summary, we have the following estimate of the norm of $u_{1}$ :

$$
\left\|u_{1}\right\|_{p} \leq C \delta\|u\|_{p}
$$

for some constant $C$ independent of $\delta$.

Now we estimate $u_{2}$. By Lemma 3.4, (a) and (4.9),

$$
\begin{aligned}
\int_{D_{i j}}\left|R_{k}(z, w)-R_{k}\left(z, z_{i j}\right)\right| d w & \leq C \int_{D_{i j}} \rho\left(w, z_{i j}\right) \frac{z_{i j n}^{k}}{\left|z-\bar{z}_{i j}\right|^{n+k}} d w \\
& \leq C \delta\left|D_{i j}\right| \frac{z_{i j n}^{k}}{\left|z-\bar{z}_{i j}\right|^{n+k}} \\
& \leq C \delta\left|D_{i j}\right| \frac{a_{i n}^{k}}{\left|z-\bar{a}_{i}\right|^{n+k}}
\end{aligned}
$$


Also, by (a) and Lemma 3.6,

$$
\left|u\left(z_{i j}\right)\right| \leq \frac{C}{\left|D_{i j}\right|} \int_{D_{i j}}|u(w)| d w .
$$

Combining these observations, we have by (b) and Hölder's inequality

$$
\begin{aligned}
\left|u_{2}(z)\right| & \leq C \delta \sum_{i, j} \frac{a_{i n}^{k}}{\left|z-\bar{a}_{i}\right|^{n+k}} \int_{D_{i j}}|u(w)| d w \\
& \leq C \delta \sum_{i} \frac{a_{i n}^{k}}{\left|z-\bar{a}_{i}\right|^{n+k}} \int_{E_{i}^{*}}|u(w)| d w \\
& \leq C \delta \sum_{i}\left\{\int_{E_{i}^{*}}|u(w)|^{p} d w\right\}^{1 / p} \frac{\left|E_{i}^{*}\right|^{1 / q} a_{i n}^{k}}{\left|z-\bar{a}_{i}\right|^{n+k}} \\
& \leq C \delta \sum_{i} \lambda_{i} a_{i n}^{n / q} \frac{a_{i n}^{k}}{\left|z-\bar{a}_{i}\right|^{n+k}}
\end{aligned}
$$

where

$$
\lambda_{i}=\left\{\int_{E_{i}^{*}}|u(w)|^{p} d w\right\}^{1 / p}
$$

Note that, by (4.9),

$$
\frac{a_{i n}^{k}}{\left|z-\bar{a}_{i}\right|^{n+k}}=\left|E_{i}^{*}\right|^{-1} \int_{E_{i}^{*}} \frac{a_{i n}^{k}}{\left|z-\bar{a}_{i}\right|^{n+k}} d s \leq C\left|E_{i}^{*}\right|^{-1} \int_{E_{i}^{*}} \frac{s_{n}^{k}}{|z-\bar{s}|^{n+k}} d s
$$

and hence, by (4.12),

$$
\left|u_{2}(z)\right| \leq \Phi_{k}\left[C \delta \sum \lambda_{i} a_{i n}^{n / q}\left|E_{i}^{*}\right|^{-1} \chi_{i}\right](z)
$$

where $\Phi_{k}$ is the operator defined in Lemma 4.4 and $\chi_{i}$ denotes the characteristic function of $E_{i}^{*}$. Now, the $L^{p}$-boundedness of $\Phi_{k}$ gives

$$
\begin{aligned}
\left\|u_{2}\right\|_{p}^{p} & \leq C \delta^{p} \sum\left|\lambda_{i}\right|^{p} a_{i n}^{n(p-1)}\left|E_{i}^{*}\right|^{1-p} \\
& \leq C \delta^{p} \sum\left|\lambda_{i}\right|^{p} \\
& =C \delta^{p} \sum \int_{E_{i}^{*}}|u(w)|^{p} d w \\
& \leq C \delta^{p} \int_{H}|u(w)|^{p} d w .
\end{aligned}
$$


The last inequality of the above holds by Lemma 4.2. Here, the constant $C$ is independent of $\delta$. Thus we have the following estimate of the norm of $u_{2}$ :

$$
\left\|u_{2}\right\|_{p} \leq C \delta\|u\|_{p}
$$

for some constant $C$ independent of $\delta$. We therefore conclude from (4.11) and (4.13) that

$$
\left\|Q_{k} T-I\right\| \leq C \delta
$$

where $I$ denotes the identity on $b^{p}$ for some constant $C$ independent of $\delta$. Accordingly, $Q_{k} T$ is invertible for all $\delta$ sufficiently small. For such $\delta$, set $P_{k}=T\left(Q_{k} T\right)^{-1}$. The proof is complete.

The $b^{1}$-representation theorem takes exactly the same form as the above $b^{p}$-representation theorem except for the restriction $k \geq 1$. The proof is also almost the same and thus omitted.

THEOREM 4.7. Let $k \geq 1$ be an integer. Then there exists a positive number $\delta_{0}$ with the following property: Let $\left\{z_{m}\right\}$ be a $\delta$-lattice with $\delta<\delta_{0}$ and let $Q_{k}: l^{1} \rightarrow b^{1}$ be the associated linear operator as in (4.6). Then there is a bounded linear operator $P_{k}: b^{1} \rightarrow l^{1}$ such that $Q_{k} P_{k}$ is the identity on $b^{1}$. In particular, $\left\{z_{m}\right\}$ is a $b^{1}$-representing sequence of order $k$.

The above proof of Theorem 4.6 also gives some other information on norm representation of $b^{p}$-functions. Namely, one can conclude from (4.8) and (4.10)

$$
\int_{H}|u(w)|^{p} d w \approx \sum\left|u\left(z_{m}\right)\right|^{p} z_{m n}^{n}
$$

as $u$ ranges over $b^{p}$. Such norm representation remains true with normal derivatives of an arbitrary order.

Proposition 4.8. Let $1 \leq p<\infty$ and $k \geq 0$ be an integer. If $\left\{z_{m}\right\}$ is a $\delta$-lattice with $\delta$ sufficiently small, then

$$
\int_{H}|u(w)|^{p} d w \approx \sum\left|D^{k} u\left(z_{m}\right)\right|^{p} z_{m n}^{n+p k}
$$

as $u$ ranges over $b^{p}$. 
Proof. Let $u \in b^{p}$. We continue to use the notations defined in the proof of Theorem 4.6. Note that

$$
\begin{aligned}
& \int_{H} w_{n}^{p k}\left|D^{k} u(w)\right|^{p} d w-\sum_{i, j} z_{i j n}^{p k}\left|D^{k} u\left(z_{i j}\right)\right|^{p}\left|D_{i j}\right| \\
& \leq C \sum_{i, j} \int_{D_{i j}}\left|w_{n}^{k} D^{k} u(w)-z_{i j n}^{k} D^{k} u\left(z_{i j}\right)\right|^{p} d w \\
& \leq C \sum_{i, j} \int_{D_{i j}}\left|w_{n}^{k}-z_{i j n}^{k}\right|^{p}\left|D^{k} u(w)\right|^{p} d w \\
& \quad+C \sum_{i, j} \int_{D_{i j}} z_{i j n}^{p k}\left|D^{k} u(w)-D^{k} u\left(z_{i j}\right)\right|^{p} d w \\
& =\mathrm{I}+\mathrm{II} .
\end{aligned}
$$

It follows from (a) and Lemma 3.2 that $\left|1-z_{i j n} / w_{n}\right| \leq 2 \delta /(1-\delta)$ for $w \in D_{i j}$ and therefore

$$
\mathrm{I} \leq C \delta^{p} \sum_{i, j} \int_{D_{i j}} w_{n}^{p k}\left|D^{k} u(w)\right|^{p} d w=C \delta^{p} \int_{H} w_{n}^{p k}\left|D^{k} u(w)\right|^{p} d w
$$

for some constant $C$ independent of $\delta$ small. Also, as in the proof of (4.10), we have

$$
\begin{aligned}
\sum_{j=1}^{N_{i}} z_{i j n}^{p k} \int_{D_{i j}}\left|D^{k} u(w)-D^{k} u\left(z_{i j}\right)\right|^{p} d w & \leq C \delta^{n+p} \sum_{j=1}^{N_{i}} \int_{E_{i}^{*}} w_{n}^{p k}\left|D^{k} u(w)\right|^{p} d w \\
& \leq C \delta^{p} \int_{E_{i}^{*}} w_{n}^{p k}\left|D^{k} u(w)\right|^{p} d w,
\end{aligned}
$$

so that

$$
\mathrm{II} \leq C \delta^{p} \sum_{i} \int_{E_{i}^{*}} w_{n}^{p k}\left|D^{k} u(w)\right|^{p} d w \leq C \delta^{p} \int_{H} w_{n}^{p k}\left|D^{k} u(w)\right|^{p} d w .
$$

Here, the constant $C$ is also independent of $\delta$ small. It follows that

$$
\begin{aligned}
\left(1-C \delta^{p}\right) \int_{H} w_{n}^{p k}\left|D^{k} u(w)\right|^{p} d w & \leq \sum_{i, j} z_{i j n}^{p k}\left|D^{k} u\left(z_{i j}\right)\right|^{p}\left|D_{i j}\right| \\
& \leq C \sum_{i, j}\left|D^{k} u\left(z_{i j}\right)\right|^{p} z_{i j n}^{n+p k}
\end{aligned}
$$


Now, since $C$ is a constant independent of $\delta$ small, we see from (2.7) that one inequality holds for $\delta$ sufficiently small. The other inequality easily follows from Lemma 3.6.

Remark 2. Proposition 4.8 yields an interesting consequence. Namely, if the normal derivative of a $b^{p}$-function $u$ vanishes on a $\delta$-lattice with $\delta$ sufficiently small, then $u$ must vanish identically. This seems a new uniqueness result for harmonic Bergman functions. This property also extends to harmonic Bloch functions. See Proposition 4.12 below.

Now, we turn to the analogous representation property for the spaces $\widetilde{\mathcal{B}}$ and $\widetilde{\mathcal{B}}_{0}$.

Representation on $\widetilde{\mathcal{B}}$ and $\widetilde{\mathcal{B}_{0}}$

Recall that we also have the reproducing formula (2.9) for harmonic Bloch functions. Thus, a consideration of approximating Riemann sum leads us to a similar definition of representing sequences for the spaces $\widetilde{\mathcal{B}}$ and $\widetilde{\mathcal{B}}_{0}$. Let $\left\{z_{m}\right\}$ be a sequence in $H$ and $k \geq 0$ be an integer. For $\left(\lambda_{m}\right) \in l^{\infty}$, let

$$
\widetilde{Q}_{k}\left(\lambda_{m}\right)(z)=\sum \lambda_{m} z_{m n}^{n} \widetilde{R}_{k}\left(z, z_{m}\right) \quad(z \in H) .
$$

We say that $\left\{z_{m}\right\}$ is a $\widetilde{\mathcal{B}}$-representing sequence of order $k$ if $\widetilde{Q}_{k}\left(l^{\infty}\right)=\widetilde{\mathcal{B}}$. We also say that $\left\{z_{m}\right\}$ is a $\widetilde{\mathcal{B}}_{0}$-representing sequence of order $k$ if $\widetilde{Q}_{k}\left(c_{0}\right)=\widetilde{\mathcal{B}}_{0}$.

As in the case of $b^{p}$-representation, we begin with an observation that a separated sequence represents a part of the whole space.

Proposition 4.9. Let $k \geq 0$ be an integer and suppose $\left\{z_{m}\right\}$ is a $\delta$ separated sequence. Let $\widetilde{Q}_{k}$ be the associated operator as in (4.14). Then, $\widetilde{Q}_{k}: l^{\infty} \rightarrow \widetilde{\mathcal{B}}$ is bounded. In addition, $\widetilde{Q}_{k}$ maps $c_{0}$ into $\widetilde{\mathcal{B}}_{0}$.

Proof. Note $\widetilde{R}_{k}(z, w)=(-2)^{k}(k !)^{-1} w_{n}^{k} D_{w_{n}}^{k} \widetilde{R}(z, w)$. We first show that the series in (4.14) converges uniformly on compact subsets of $H$. Let $K$ be a compact subset of $H$. Then, by (2.8) and Lemma 3.3, there exists a constant $C=C(K)$ such that

$$
|\widetilde{R}(z, w)| \leq \frac{C}{\left|w-\bar{z}_{0}\right|^{n+1}}
$$

for all $z \in K$ and $w \in H$. Thus, it follows from Lemma 3.6 that

$$
z_{m n}^{n}\left|\widetilde{R}_{k}\left(z, z_{m}\right)\right| \leq C \int_{E_{\delta}\left(z_{m}\right)}|\widetilde{R}(z, w)| d w \leq C \int_{E_{\delta}\left(z_{m}\right)} \frac{d w}{\left|w-\bar{z}_{0}\right|^{n+1}}
$$


for all $z \in K$. Since $\left|w-\bar{z}_{0}\right|^{-(n+1)}$ is integrable, it follows that the series in (4.14) converges uniformly on $K$. Note that $D_{z_{n}} \widetilde{R}(z, w)=D_{z_{n}} R(z, w)$. Now, after differentiating term by term, apply Lemma 3.6, (3.7), Lemma 3.8, to obtain

$$
\begin{aligned}
D \widetilde{Q}\left(\lambda_{m}\right)(z) & \leq C \sum\left|\lambda_{m}\right| z_{m n}^{n+k}\left|D_{z_{m n}}^{k} D_{z_{n}} R\left(z, z_{m}\right)\right| \\
& \leq C\left\|\left(\lambda_{m}\right)\right\|_{\infty} \sum \int_{E_{\delta}\left(z_{m}\right)}\left|D_{z_{n}} R(z, w)\right| d w \\
& \leq C\left\|\left(\lambda_{m}\right)\right\|_{\infty} \int_{H} \frac{d w}{|z-\bar{w}|^{n+1}} \\
& \leq C z_{n}^{-1}\left\|\left(\lambda_{m}\right)\right\|_{\infty} .
\end{aligned}
$$

In other words, by (2.11),

$$
\left\|\widetilde{Q}\left(\lambda_{m}\right)\right\|_{\mathcal{B}} \leq C\left\|\left(\lambda_{m}\right)\right\|_{\infty},
$$

which shows the first part of the proposition.

Next, assume $\left(\lambda_{m}\right) \in c_{0}$ and put

$$
u_{N}(z)=\sum_{j=1}^{N} \lambda_{j} z_{j n}^{n} \widetilde{R}_{k}\left(z, z_{j}\right) \quad(N=1,2, \cdots)
$$

Since $D_{z_{n}} \widetilde{R}_{k}(z, w)=-(k+1) 2^{-1} w_{n}^{-1} R_{k+1}(z, w)$, it is easily checked $\widetilde{R}_{k}(\cdot, w)$ $\in \widetilde{\mathcal{B}}_{0}$ for each fixed $w$ and therefore $u_{N} \in \widetilde{\mathcal{B}}_{0}$ for all $N$. By (4.15), for every $N$, we have

$$
\left\|\widetilde{Q}_{k}\left(\lambda_{m}\right)-u_{N}\right\|_{\mathcal{B}} \leq C\left(\sup _{j \geq N+1}\left|\lambda_{j}\right|\right)
$$

so that $u_{N}$ converges to $\widetilde{Q}_{k}\left(\lambda_{m}\right)$ in norm. Hence $\widetilde{Q}_{k}\left(\lambda_{m}\right) \in \widetilde{\mathcal{B}}_{0}$. The proof is complete.

Having Proposition 4.9, one can modify the proof of Theorem 4.6 to obtain a similar $\widetilde{\mathcal{B}}$-representation theorem. As a substitute for integral estimates in the proof of Theorem 4.6, we need the following lemma.

LEMMA 4.10. Let $k \geq 1$ be an integer. Then

$$
\left|z_{n}^{k} D^{k} u(z)-w_{n}^{k} D^{k} u(w)\right| \leq C \rho(z, w)\|u\|_{\mathcal{B}}
$$

for all $z, w \in H$ and $u \in \widetilde{\mathcal{B}}$. 
Proof. Let $u \in \widetilde{\mathcal{B}}$ and fix $z, w \in H$. We may assume $\rho(z, w)<$ $1 / 2$. By passing differentiation through the integral sign in the generalized reproducing formula $(2.10)$, we have

$$
\begin{array}{rl}
z_{n}^{k} D^{k} & u(z)-w_{n}^{k} D^{k} u(w) \\
& =-2 \int_{H} s_{n}[D u(s)]\left(z_{n}^{k} D_{z_{n}}^{k} \widetilde{R}(z, s)-w_{n}^{k} D_{w_{n}}^{k} \widetilde{R}(w, s)\right) d s \\
& =k !(-2)^{1-k} \int_{H} s_{n}[D u(s)]\left(R_{k}(s, z)-R_{k}(s, w)\right) d s
\end{array}
$$

and therefore, after taking absolute values, we obtain from Lemma 3.4 and Lemma 3.8 that

$$
\begin{aligned}
\left|z_{n}^{k} D^{k} u(z)-w_{n}^{k} D^{k} u(w)\right| & \leq C \rho(z, w)\|u\|_{\mathcal{B}} \int_{H} \frac{z_{n}^{k}}{|z-\bar{s}|^{n+k}} d s \\
& \leq C \rho(z, w)\|u\|_{\mathcal{B}}
\end{aligned}
$$

which completes the proof.

The following is the limiting version of the $b^{p}$-representation theorem (Theorem 4.6). For a similar result on the ball, see Theorem $3^{\prime}$ of [5].

THEOREM 4.11. Let $k \geq 0$ be an integer. Then there exists a positive number $\delta_{0}$ with the following property: Let $\left\{z_{m}\right\}$ be a $\delta$-lattice with $\delta<\delta_{0}$ and let $\widetilde{Q}_{k}: l^{\infty} \rightarrow \widetilde{\mathcal{B}}$ be the associated linear operator as in (4.14). Then there exists a bounded linear operator $\widetilde{P}_{k}: \widetilde{\mathcal{B}} \rightarrow l^{\infty}$ such that $\widetilde{Q}_{k} \widetilde{P}_{k}$ is the identity on $\widetilde{\mathcal{B}}$. Moreover, $\widetilde{P}_{k}$ maps $\widetilde{\mathcal{B}}_{0}$ into $c_{0}$. In particular, $\left\{z_{m}\right\}$ is a both $\widetilde{\mathcal{B}}$-representing and $\widetilde{\mathcal{B}}_{0}$-representing sequence of order $k$.

Proof. The proof is similar to that of Theorem 4.6. Any unexplained notation will have the same meaning as in the proof Theorem 4.6. For $u \in \widetilde{\mathcal{B}}$, let $\widetilde{T} u$ denote the sequence whose components are $z_{i j n}^{1-n} D u\left(z_{i j}\right)\left|D_{i j}\right|$. Then, by (a), $\widetilde{T}: \widetilde{\mathcal{B}} \rightarrow l^{\infty}$ is bounded. Since the sequence $\left\{z_{m}\right\}$ is separated, we have $z_{m} \rightarrow \partial H \cup\{\infty\}$. Thus $\widetilde{T}$ maps $\widetilde{\mathcal{B}}_{0}$ into $c_{0}$. It follows that $\widetilde{Q}_{k} \widetilde{T}$ is bounded on $\widetilde{\mathcal{B}}$ and maps $\widetilde{\mathcal{B}}_{0}$ into itself by Proposition 4.9. As in the proof of Theorem 4.6 , it suffices to show $\widetilde{Q}_{k} \widetilde{T}$ is invertible on $\widetilde{\mathcal{B}}$ for all $\delta$ sufficiently small.

Note that

$$
\widetilde{Q}_{k} \tilde{T} u(z)=\sum z_{i j n} D u\left(z_{i j}\right) \widetilde{R}_{k}\left(z, z_{i j}\right)\left|D_{i j}\right|
$$


and $\widetilde{R}_{k}\left[w_{n} D u(w)\right]=-(k+1) u / 2$ by the generalized reproducing property (2.10). Hence we can decompose $-(k+1) u / 2-\widetilde{Q}_{k} \widetilde{T} u=u_{1}+u_{2}$ where

$$
\begin{aligned}
& u_{1}(z)=\widetilde{R}_{k}\left[\sum\left(w_{n} D u(w)-z_{i j n} D u\left(z_{i j}\right)\right) \chi_{i j}\right](z) \\
& u_{2}(z)=\sum z_{i j n} D u\left(z_{i j}\right) \int_{D_{\imath \jmath}} \widetilde{R}_{k}(z, w)-\widetilde{R}_{k}\left(z, z_{i j}\right) d w .
\end{aligned}
$$

For $u_{1}$, since $\widetilde{R}_{k}: L^{\infty} \rightarrow \widetilde{\mathcal{B}}$ is bounded, we have, by Lemma 4.10 and (a), the following norm estimate of $u_{1}$ :

$$
\begin{aligned}
\left\|u_{1}\right\|_{\mathcal{B}} & =\left\|\widetilde{R}_{k}\left[\sum\left(w_{n} D u(w)-z_{i j n} D u\left(z_{i j}\right)\right) \chi_{i j}\right]\right\|_{\mathcal{B}} \\
& \leq C\left\|\sum\left(w_{n} D u(w)-z_{i j n} D u\left(z_{i j}\right)\right) \chi_{i j}\right\|_{\infty} \\
& \leq C\|u\|_{\mathcal{B}}\left(\sup _{i, j} \sup _{w \in D_{i j}} \rho\left(w, z_{i j n}\right)\right) \\
& \leq C \delta\|u\|_{\mathcal{B}} .
\end{aligned}
$$

Note that the above constant $C$ is independent of $\delta$.

Differentiating term by term and then differentiating under the integral sign, we have

$$
\begin{aligned}
D u_{2}(z) & =\sum z_{i j n} D u\left(z_{i j}\right) \int_{D_{i j}} D_{z_{n}} \widetilde{R}_{k}(z, w)-D_{z_{n}} \widetilde{R}_{k}\left(z, z_{i j}\right) d w \\
& =-\frac{k+1}{2} \sum z_{i j n} D u\left(z_{i j}\right) \int_{D_{i j}} w_{n}^{-1} R_{k+1}(z, w)-z_{i j n}^{-1} R_{k+1}\left(z, z_{i j}\right) d w
\end{aligned}
$$

After taking absolute values, we see from (a) and Lemma 3.4

$$
\begin{aligned}
\left|D u_{2}(z)\right| & \leq C\|u\|_{\mathcal{B}} \sum \int_{D_{i j}} \rho\left(w, z_{i j}\right) \frac{w_{n}^{k}}{|z-\bar{w}|^{n+k+1}} d w \\
& \leq C \delta\|u\|_{\mathcal{B}} \sum \int_{D_{i j}} \frac{d w}{|z-\bar{w}|^{n+1}} \\
& =C \delta\|u\|_{\mathcal{B}} \int_{H} \frac{d w}{|z-\bar{w}|^{n+1}} \\
& \leq C \delta z_{n}^{-1}\|u\|_{\mathcal{B}}
\end{aligned}
$$

where the last inequality holds by Lemma 3.8. This, together with (2.11), yields the following norm estimate of $u_{2}$ :

$$
\left\|u_{2}\right\|_{\mathcal{B}} \leq C \delta\|u\|_{\mathcal{B}}
$$


Here, the constant $C$ is also independent of $\delta$. Now, letting $I$ denote the identity on $\widetilde{\mathcal{B}}$, we conclude from (4.16) and (4.17) that

$$
\left\|\widetilde{Q}_{k} \widetilde{T}+\frac{k+1}{2} \mathrm{I}\right\| \leq C \delta
$$

for some constant $C$ independent of $\delta$. Accordingly, $\widetilde{Q}_{k} \widetilde{T}$ is invertible on $\widetilde{\mathcal{B}}$ for all $\delta$ sufficiently small, as desired. The proof is complete.

Lemma 4.10 also yields the following result for $\widetilde{\mathcal{B}}$ analogous to Proposition 4.8 .

Proposition 4.12. Let $k \geq 1$ be an integer. If $\left\{z_{m}\right\}$ is a $\delta$-lattice with $\delta$ sufficiently small, then

$$
\|u\|_{\mathcal{B}} \approx \sup _{m} z_{m n}^{k}\left|D^{k} u\left(z_{m}\right)\right|
$$

as $u$ ranges over $\tilde{\mathcal{B}}$.

Proof. Let $\left\{z_{m}\right\}$ be a $\delta$-lattice with $\delta<1 / 8$ and let $\left\{D_{i j}\right\}$ be the pairwise disjoint covering of $H$ associated with $\left\{z_{m}\right\}$ as in Lemma 4.1. Then, for $u \in \widetilde{\mathcal{B}}$ and for $w \in D_{i j}$, we have by (a) and Lemma 4.10

$$
\left|w_{n}^{k} D^{k} u(w)-z_{i j}^{k} D^{k} u\left(z_{i j}\right)\right| \leq C \delta\|u\|_{\mathcal{B}}
$$

and hence

$$
\sup _{w \in D_{i j}} w_{n}^{k}\left|D^{k} u(w)\right|-C \delta\|u\|_{\mathcal{B}} \leq z_{i j n}^{k}\left|D^{k} u\left(z_{i j}\right)\right|
$$

Taking supremum over all $i, j$, we have from (2.11)

$$
C\|u\|_{\mathcal{B}}-C \delta\|u\|_{\mathcal{B}} \leq \sup _{i, j} z_{i j n}^{k}\left|D^{k} u\left(z_{i j}\right)\right|
$$

which implies the proposition.

A result for holomorphic Bloch functions (on the disk) similar to the above proposition can be found in [2]. 


\section{$\S 5$. Interpolations}

Consider a $\delta$-separated sequence $\left\{z_{m}\right\}$. Let $k \geq 0$ be an integer and $1 \leq p<\infty$. By Lemma 3.6 we have

$$
\sum z_{m n}^{n+p k}\left|D^{k} u\left(z_{m}\right)\right|^{p} \leq C \sum \int_{E_{\delta}\left(z_{m}\right)}|u|^{p} d V \leq C \int_{H}|u|^{p} d V
$$

for all $u \in b^{p}$. In other words, if we let $T_{k} u$ denote the sequence whose $m$-th component is $z_{m n}^{n / p+k} D^{k} u\left(z_{m}\right)$, then the operator $T_{k}: b^{p} \rightarrow l^{p}$ is bounded. However, having seen representation theorems in the previous section, one cannot expect that such $T_{k}$ is onto in general. In fact, by Proposition 4.8, $T_{k}$ is one-to-one for $\delta$ sufficiently small and $T_{k}$ cannot be expected to be an isomorphism in general. This "interpolating" operator $T_{k}$ is closely related with the "representing" operator $Q_{k}$ (studied in the previous section) in the sense that, for $1<p<\infty$ and its conjugate exponent $q$, the adjoint of $Q_{k}: l^{q} \rightarrow b^{q}$ can be identified (up to a constant factor) with $T_{k}: b^{p} \rightarrow l^{p}$ under the standard integral pairing if the underlying sequence is separated. For the duality $\left(b^{p}\right)^{*}=b^{q}$, see [8].

In view of interpolation results of [1], [9] for holomorphic Bergman functions on various domains, a good candidate condition for "onto" is "sufficient separation." In this section we prove that the same phenomenon persists to hold on the setting of the present paper. As a limiting case, we will also consider interpolation on the spaces $\widetilde{\mathcal{B}}, \widetilde{\mathcal{B}}_{0}$ and get similar results. In the holomorphic case, such interpolation property on the Bloch space was studied in [2], [4].

\section{Interpolation on $b^{p}$}

Let $\left\{z_{m}\right\}$ be a sequence in $H$. Let $k \geq 0$ be an integer and $1 \leq p<\infty$. For $u \in b^{p}$, let $T_{k} u$ denote the sequence of complex numbers defined by

$$
T_{k} u=\left(z_{m n}^{n / p+k} D^{k} u\left(z_{m}\right)\right) .
$$

Thus $T_{k}$ is a linear operator taking $b^{p}$ into the space of all sequences of complex numbers. We say that $\left\{z_{m}\right\}$ is a $b^{p}$-interpolating sequence of order $k$ if $T_{k}\left(b^{p}\right)=l^{p}$.

We first show that separation is necessary for $b^{p}$-interpolation. We need a couple of lemmas.

LEMMA 5.1. Let $\left\{z_{m}\right\}$ be a $b^{p}$-interpolating sequence of order $k$. Let $T_{k}$ denote the associated operator as in (5.1). Then, $T_{k}: b^{p} \rightarrow l^{p}$ is bounded. 
Proof. Assume $u_{j} \rightarrow u$ in $b^{p}$ and $T_{k} u_{j} \rightarrow\left(\lambda_{m}\right)$ in $l^{p}$. By the closed graph theorem, we only need to show $T_{k} u=\left(\lambda_{m}\right)$. By Lemma 3.6, for any positive integer $N$, we have

$$
\begin{aligned}
& \left\|T_{k} u-\left(\lambda_{m}\right)\right\|_{p}^{p} \\
& =\sum_{1}^{\infty}\left|z_{m n}^{n / p+k} D^{k} u\left(z_{m}\right)-\lambda_{m}\right|^{p} \\
& \leq C \sum_{1}^{N} z_{m n}^{n+p k}\left|D^{k} u\left(z_{m}\right)-D^{k} u_{j}\left(z_{m}\right)\right|^{p}+C \sum_{1}^{N}\left|z_{m n}^{n / p+k} D^{k} u_{j}\left(z_{m}\right)-\lambda_{m}\right|^{p} \\
& \quad+\sum_{N+1}^{\infty}\left|z_{m n}^{n / p+k} D^{k} u\left(z_{m}\right)-\lambda_{m}\right|^{p} \\
& \leq C N\left\|u-u_{j}\right\|_{p}^{p}+C\left\|T_{k} u_{j}-\left(\lambda_{m}\right)\right\|_{p}^{p}+\sum_{N+1}^{\infty}\left|z_{m n}^{n / p+k} D^{k} u\left(z_{m}\right)-\lambda_{m}\right|^{p} .
\end{aligned}
$$

Now, taking first the limit $j \rightarrow \infty$ and then $N \rightarrow \infty$, we obtain $T_{k} u=\left(\lambda_{m}\right)$. The proof is complete.

The following can be viewed as a $b^{p}$-version of Lemma 4.10 .

LemMA 5.2. Let $1 \leq p<\infty$ and $k \geq 0$ be an integer. Then

$$
\left|z_{n}^{n / p+k} D^{k} u(z)-w_{n}^{n / p+k} D^{k} u(w)\right| \leq C \rho(z, w)\|u\|_{p}
$$

for all $z, w \in H$ and $u \in b^{p}$.

Proof. Let $u \in b^{p}$ and fix $z, w \in H$. By Lemma 3.6, we may assume $\rho(z, w)<1 / 2$. First, by the reproducing formula (2.5) and differentiation under the integral sign, we have

$$
\begin{aligned}
& z_{n}^{n / p+k} D^{k} u(z)-w_{n}^{n / p+k} D^{k} u(w) \\
& \quad=k !(-2)^{-k} \int_{H} u(s)\left(z_{n}^{n / p} R_{k}(s, z)-w_{n}^{n / p} R_{k}(s, w)\right) d s .
\end{aligned}
$$

Note that

$$
\left|z_{n}^{n / p} R_{k}(s, z)-w_{n}^{n / p} R_{k}(s, w)\right| \leq C \rho(z, w) \frac{z_{n}^{n / p+k}}{|z-\bar{s}|^{n+k}}
$$


by Lemma 3.4. Since $z_{n}^{n+k}|z-\bar{s}|^{-(n+k)} \leq 1$, this proves the lemma for $p=1$. For $p>1$, after taking absolute values, apply Hölder's inequality and then Lemma 3.8 to obtain

$$
\begin{aligned}
\mid z_{n}^{n / p+k} D^{k} u(z) & -w_{n}^{n / p+k} D^{k} u(w) \mid \\
& \leq C \rho(z, w) \int_{H}|u(s)| \frac{z_{n}^{n / p+k}}{|z-\bar{s}|^{n+k}} d s \\
& \leq C \rho(z, w)\|u\|_{p}\left\{\int_{H} \frac{z_{n}^{q(n+k)-n}}{|z-\bar{s}|^{q(n+k)}} d s\right\}^{1 / q} \\
& \leq C \rho(z, w)\|u\|_{p}
\end{aligned}
$$

where $q$ is the conjugate exponent of $p$. The proof is complete.

Proposition 5.3. Every $b^{p}$-interpolating sequence of order $k$ is separated.

Proof. Let a $b^{p}$-interpolating sequence of order $k$ be given. Then, by Lemma 5.1, the associated linear operator $T_{k}: b^{p} \rightarrow l^{p}$ is bounded and onto. Hence, by the open mapping theorem, one can find a uniformly bounded sequence $u_{j}$ in $b^{p}$ such that $T_{k} u_{j}$ is the sequence whose components are all 0 except for the $j$-th component 1 . Now, the proposition follows from Lemma 5.2.

Before proceeding to the proof that "sufficient separation" is sufficient for $b^{p}$-interpolation, we first prove a lemma.

LemMA 5.4. Let $1<p<\infty$ and $k \geq 0$ be an integer. Suppose $\left\{z_{m}\right\}$ is a $\delta$-separated sequence. Then, for $\left(\lambda_{m}\right) \in l^{p}$, we have

$$
\left|\sum \lambda_{m} z_{m n}^{n / q} R_{k}\left(z_{m}, w\right)\right|^{p} \leq C \delta^{-n(p-1)} w_{n}^{-1 / q} \sum\left|\lambda_{m}\right|^{p} z_{m n}^{1 / q}\left|R_{k}\left(z_{m}, w\right)\right|
$$

for $w \in H$ where $q$ is the conjugate exponent of $p$. The constant $C$ is independent of $\delta$.

Proof. Note that

$$
\left|\lambda_{m}\right| z_{m n}^{n / q}\left|R_{k}\left(z_{m}, w\right)\right|=\left|\lambda_{m}\right| z_{m n}^{1 / p q}\left|R_{k}\left(z_{m}, w\right)\right|^{1 / p} \cdot z_{m n}^{n / q} z_{m n}^{-1 / p q}\left|R_{k}\left(z_{m}, w\right)\right|^{1 / q} .
$$

Thus, by Hölder's inequality, the left side of (5.2) is less than or equal to

$$
\sum\left|\lambda_{m}\right|^{p} z_{m n}^{1 / q}\left|R_{k}\left(z_{m}, w\right)\right|\left(\sum z_{m n}^{n-1 / p}\left|R_{k}\left(z_{m}, w\right)\right|\right)^{p / q}
$$


Note that $1 / 3<z_{m n} / s_{n}<3$ for $s \in E_{\delta / 2}\left(z_{m}\right)$ by Lemma 3.2. Thus, by Lemma 3.6 and (3.7), the sum inside the parenthesis of the above is less than or equal to

$$
\begin{aligned}
C \delta^{-n} \sum z_{m n}^{-1 / p} & \int_{E_{\delta / 2}\left(z_{m}\right)}\left|R_{k}(s, w)\right| d s \\
& \leq C \delta^{-n} \sum \int_{E_{\delta / 2}\left(z_{m}\right)} \frac{w_{n}^{k} s_{n}^{-1 / p}}{|s-\bar{w}|^{n+k}} d s \\
& \leq C \delta^{-n} \int_{H} \frac{w_{n}^{k} s_{n}^{-1 / p}}{|s-\bar{w}|^{n+k}} d s \\
& \leq C \delta^{-n} \int_{H} \frac{s_{n}^{-1 / p}}{|s-\bar{w}|^{n}} d s \\
& \leq C \delta^{-n} w_{n}^{-1 / p}
\end{aligned}
$$

where the last inequality holds by Lemma 3.9. Here, the constant $C=$ $C(n, p, k)$ is independent of $\delta$. The proof is complete.

Now we prove our $b^{p}$-interpolation theorem. Recall that the interpolation is the dual property of representation in a certain sense. Thus, even though we do not use any duality argument here, it is not too surprising to see that the following $b^{p}$-interpolation theorem takes a very similar form to the $b^{p}$-representation theorem of the previous section. Duality does provide some motivation for the proof, however.

ThEOREM 5.5. Let $1 \leq p<\infty$ and $k \geq 0$ be an integer. Then there exists a positive number $\delta_{0}$ with the following property: Let $\left\{z_{m}\right\}$ be a $\delta$ separated sequence with $\delta>\delta_{0}$ and let $T_{k}: b^{p} \rightarrow l^{p}$ be the associated linear operator as in (5.1). Then there is a bounded linear operator $S_{k}: l^{p} \rightarrow b^{p}$ such that $T_{k} S_{k}$ is the identity on $l^{p}$. In particular, $\left\{z_{m}\right\}$ is a $b^{p}$-interpolating sequence of order $k$.

Proof. Fix an integer $k \geq 0$. First, it is not hard to verify (by induction) that the function $w \mapsto k !(-2)^{-k} w_{n}^{n} R_{k}(w, w)$ is constant on $H$. We will let $c_{k}(\neq 0)$ denote this constant (one may compute $c_{k}=$ $\left.4(-1)^{k}(n+k-1) ! / n \sigma_{n} 2^{n+k}(n-2) !\right)$. The proof is splitted into two cases.

The case $1<p<\infty$ : Fix $\left(\lambda_{m}\right) \in l^{p}$. Let $Q\left(\lambda_{m}\right)$ denote the function defined by

$$
Q\left(\lambda_{m}\right)(w)=\sum \lambda_{m} z_{m n}^{n / q} R\left(w, z_{m}\right) \quad(w \in H)
$$


where $q$ is the conjugate exponent of $p$. Then, by Proposition 4.5, $Q: l^{p} \rightarrow b^{p}$ is a bounded linear operator. Thus $T_{k} Q$ is bounded on $l^{p}$.

We claim that $T_{k} Q$ is invertible on $l^{p}$ for all $\delta$ sufficiently close to 1. Let $I$ denote the identity on $l^{p}$ and let $\alpha_{j}$ denote the $j$-th component of the sequence $\left(T_{k} Q-c_{k} I\right)\left(\lambda_{m}\right)$. Note that the $j$-th component of the sequence $T_{k} Q\left(\lambda_{m}\right)$ is $z_{j n}^{n / p+k} D^{k} Q\left(\lambda_{m}\right)\left(z_{j}\right)$. Thus, by term-by-term differentiation (justified by uniform convergence on compact sets), one has

$$
\alpha_{j}=k !(-2)^{-k} z_{j n}^{n / p} \sum_{m \neq j} \lambda_{m} z_{m n}^{n / q} R_{k}\left(z_{m}, z_{j}\right)
$$

Thus, by Lemma 5.4, we have

$$
\left|\alpha_{j}\right|^{p} \leq C \delta^{-n(p-1)} z_{j n}^{n-1 / q} \sum_{m \neq j}\left|\lambda_{m}\right|^{p} z_{m n}^{1 / q}\left|R_{k}\left(z_{m}, z_{j}\right)\right|
$$

so that

$$
\sum\left|\alpha_{j}\right|^{p} \leq C \delta^{-n(p-1)} \sum_{m=1}^{\infty}\left|\lambda_{m}\right|^{p} \beta_{m}
$$

where

$$
\beta_{m}=z_{m n}^{1 / q} \sum_{j \neq m} z_{j n}^{n-1 / q}\left|R_{k}\left(z_{m}, z_{j}\right)\right| .
$$

On the other hand, by Lemma 3.6, we have (as in the proof of Lemma 5.4)

$$
\begin{aligned}
\beta_{m} & \leq C \delta^{-(n+k)} z_{m n}^{1 / q} \sum_{j \neq m} z_{j n}^{-1 / q} \int_{E_{\delta / 2}\left(z_{j}\right)}\left|R\left(z_{m}, s\right)\right| d s \\
& \leq C \delta^{-(n+k)} z_{m n}^{1 / q} \sum_{j \neq m} \int_{E_{\delta / 2}\left(z_{j}\right)} s_{n}^{-1 / q}\left|R\left(z_{m}, s\right)\right| d s \\
& \leq C \delta^{-(n+k)} z_{m n}^{1 / q} \int_{H \backslash E_{\delta}\left(z_{m}\right)} \frac{s_{n}^{-1 / q}}{\left|s-\bar{z}_{m}\right|^{n}} d s \\
& =C \delta^{-(n+k)} \int_{H \backslash E_{\delta}\left(z_{0}\right)} \frac{s_{n}^{-1 / q}}{\left|s-\bar{z}_{0}\right|^{n}} d s
\end{aligned}
$$

for all $m$. Here, the constant $C$ depends only on $n, p$ and $k$. Consequently, from (5.3) and (5.4), we conclude

$$
\left\|T_{k} Q-c_{k} \mathrm{I}\right\| \leq C \delta^{-(n+k / p)}\left\{\int_{H \backslash E_{\delta}\left(z_{0}\right)} \frac{s_{n}^{-1 / q}}{\left|s-\bar{z}_{0}\right|^{n}} d s\right\}^{1 / p}
$$


for some constant $C$ independent of $\delta$. It follows from the fact that $s_{n}^{-1 / q} \mid s-$ $\left.\bar{z}_{0}\right|^{-n}$ is integrable (Lemma 3.9) that the integral of the above tends to 0 as $\delta \nearrow 1$. Thus $T_{k} Q$ is invertible on $l^{p}$ for all $\delta$ sufficiently close to 1 . For such $\delta$, put $S_{k}=Q\left(T_{k} Q\right)^{-1}$. This completes the proof for the case $1<p<\infty$.

The case $p=1$ : The proof is similar. The auxiliary operator is slightly different. Fix $\left(\lambda_{m}\right) \in l^{1}$. Let $Q\left(\lambda_{m}\right)$ denote the function defined by

$$
Q\left(\lambda_{m}\right)(w)=\sum \lambda_{m} R_{1}\left(w, z_{m}\right) \quad(w \in H) .
$$

Then, by Lemma 4.5, $Q: l^{1} \rightarrow b^{1}$ is bounded and thus $T_{k} Q$ is bounded on $l^{1}$.

We now prove $T_{k} Q$ is invertible on $l^{1}$ for all $\delta$ sufficiently close to 1 . Let $I$ denote the identity on $l^{1}$ and let $\alpha_{j}$ be the $j$-th component of the sequence $\left(T_{k} Q+2 c_{k+1} \mathrm{I}\right)\left(\lambda_{m}\right)$. Since the $j$-th component of the sequence $T_{k} Q\left(\lambda_{m}\right)$ is $z_{j n}^{n+k} D^{k} Q\left(\lambda_{m}\right)\left(z_{j}\right)$, after differentiating term-by-term, we obtain

$$
\alpha_{j}=-2 z_{j n}^{n+k} \sum_{m \neq j} \lambda_{m} z_{m n} D_{z_{j n}}^{k} D_{z_{j n}} R\left(z_{m}, z_{j}\right)
$$

and therefore by Lemma 3.6

$$
\begin{aligned}
\sum\left|\alpha_{j}\right| & \leq C \delta^{-(n+k)} \sum_{m} \sum_{j \neq m}\left|\lambda_{m}\right| \int_{E_{\delta}\left(z_{j}\right)} z_{m n}\left|D_{w_{n}} R\left(z_{m}, w\right)\right| d w \\
& \leq C \delta^{-(n+k)} \sum_{m} \sum_{j \neq m}\left|\lambda_{m}\right| \int_{E_{\delta}\left(z_{j}\right)} \frac{z_{m n}}{\left|z_{m}-\bar{w}\right|^{n+1}} d w \\
& \leq C \delta^{-(n+k)} \sum_{m}\left|\lambda_{m}\right| \int_{H \backslash E_{\delta}\left(z_{m}\right)} \frac{z_{m n}}{\left|z_{m}-\bar{w}\right|^{n+1}} d w \\
& =C \delta^{-(n+k)}\left(\sum_{m}\left|\lambda_{m}\right|\right) \int_{H \backslash E_{\delta}\left(z_{0}\right)} \frac{d w}{\left|w-\bar{z}_{0}\right|^{n+1}}
\end{aligned}
$$

where $C$ is a constant independent of $\delta$. Since $\left|w-\bar{z}_{0}\right|^{-(n+1)}$ is integrable (Lemma 3.8), one can see as above that the operator $T_{k} Q$ is invertible on $l^{1}$ for all $\delta$ sufficiently close to 1 . For such $\delta$, put $S_{k}=Q\left(T_{k} Q\right)^{-1}$. This completes the proof.

As a limiting case of $b^{p}$-interpolation, we now consider interpolations on the spaces $\widetilde{\mathcal{B}}$ and $\widetilde{\mathcal{B}_{0}}$. 
Interpolation on $\widetilde{\mathcal{B}}$ and $\widetilde{\mathcal{B}}_{0}$

Let $k \geq 1$ be an integer and let $\left\{z_{m}\right\}$ be a sequence in $H$. For $u \in \widetilde{\mathcal{B}}$, let $\widetilde{T}_{k} u$ denote the sequence of complex numbers defined by

$$
\widetilde{T}_{k} u=\left(z_{m n}^{k} D^{k} u\left(z_{m}\right)\right) .
$$

Then $\widetilde{T}_{k}: \widetilde{\mathcal{B}} \rightarrow l^{\infty}$ is clearly bounded. We say that $\left\{z_{m}\right\}$ is a $\widetilde{\mathcal{B}}$-interpolating sequence of order $k$ if $\widetilde{T}_{k}(\widetilde{\mathcal{B}})=l^{\infty}$. We also say that $\left\{z_{m}\right\}$ is a $\widetilde{\mathcal{B}}_{0^{-}}$ interpolating sequence of order $k$ if $\widetilde{T}_{k}\left(\widetilde{\mathcal{B}}_{0}\right)=c_{0}$.

As in the case of $b^{p}$-interpolation, separation turns out to be necessary for $\widetilde{\mathcal{B}}$-interpolation or $\widetilde{\mathcal{B}}_{0}$-interpolation.

Proposition 5.6. Every $\widetilde{\mathcal{B}}$-interpolating sequence of order $k$ is separated. Also, every $\widetilde{\mathcal{B}}_{0}$-interpolating sequence of order $k$ is separated.

Proof. Having Lemma 4.10, one can proceed as in the proof of Proposition 5.3.

The following theorem shows that "sufficient separation" is also sufficient for $\widetilde{\mathcal{B}}$-interpolation or $\widetilde{\mathcal{B}}_{0}$-interpolation.

THEOREM 5.7. Let $k \geq 1$ be an integer. Then there exists a positive number $\delta_{0}$ with the following property: Let $\left\{z_{m}\right\}$ be a $\delta$-separated sequence with $\delta>\delta_{0}$ and let $\widetilde{T}_{k}: \widetilde{\mathcal{B}} \rightarrow l^{\infty}$ be the associated linear operator as in (5.6). Then there exists a bounded linear operator $\widetilde{S}_{k}: l^{\infty} \rightarrow \widetilde{\mathcal{B}}$ such that $\widetilde{T}_{k} \widetilde{S}_{k}$ is the identity on $l^{\infty}$. Moreover, $\widetilde{S}_{k}$ maps $c_{0}$ into $\widetilde{\mathcal{B}}_{0}$. In particular, $\left\{z_{m}\right\}$ is a both $\widetilde{\mathcal{B}}$-interpolating and $\widetilde{\mathcal{B}}_{0}$-interpolating sequence of order $k$.

Proof. Fix a positive integer $k$ and let $\left\{z_{m}\right\}$ be a $\delta$-separated sequence. For $\left(\lambda_{m}\right) \in l^{\infty}$, let $\widetilde{Q}\left(\lambda_{m}\right)$ denote the function defined by

$$
\widetilde{Q}\left(\lambda_{m}\right)(w)=\sum \lambda_{m} z_{m n}^{n} \widetilde{R}\left(w, z_{m}\right) \quad(w \in H) .
$$

By Proposition 4.9, $\widetilde{Q}: l^{\infty} \rightarrow \widetilde{\mathcal{B}}$ is bounded and $\widetilde{Q}$ maps $c_{0}$ into $\widetilde{\mathcal{B}_{0}}$. Since the sequence $\left\{z_{m}\right\}$ is separated, we have $z_{m} \rightarrow \partial H \cup\{\infty\}$ and thus $\widetilde{T}_{k}$ maps $\widetilde{\mathcal{B}}_{0}$ into $c_{0}$. It follows that $\widetilde{T}_{k} \widetilde{Q}$ is bounded on $l^{\infty}$ and maps $c_{0}$ into itself. As in the proof of Theorem 5.5, it remains to show $\widetilde{T}_{k} \widetilde{Q}$ is invertible on $l^{\infty}$ for all $\delta$ sufficiently close to 1 . 
Let $c_{k}$ be the constant defined in the proof of Theorem 5.5 and let $I$ denote the identity on $l^{\infty}$. Since the $j$-th component of the sequence $\widetilde{T}_{k} \widetilde{Q}\left(\lambda_{m}\right)$ is $z_{j n}^{k} D^{k} \widetilde{Q}\left(\lambda_{m}\right)\left(z_{j}\right)$, term-by-term differentiation yields

$$
\alpha_{j}=k !(-2)^{-k} \sum_{m \neq j} \lambda_{m} z_{m n}^{n} R_{k}\left(z_{m}, z_{j}\right)
$$

where $\alpha_{j}$ denotes the $j$-th component of the sequence $\left(\widetilde{T}_{k} \widetilde{Q}-c_{k} \mathrm{I}\right)\left(\lambda_{m}\right)$. Thus, by Lemma 3.6 and (3.7),

$$
\begin{aligned}
\left|\alpha_{j}\right| & \leq C \delta^{-n}\left\|\left(\lambda_{m}\right)\right\|_{\infty} \sum_{m \neq j} \int_{E_{\delta}\left(z_{m}\right)}\left|R_{k}\left(z, z_{j}\right)\right| d z \\
& \leq C \delta^{-n}\left\|\left(\lambda_{m}\right)\right\|_{\infty} \sum_{m \neq j} \int_{E_{\delta}\left(z_{m}\right)} \frac{z_{j n}^{k}}{\left|z-\bar{z}_{j}\right|^{n+k}} d z \\
& \leq C \delta^{-n}\left\|\left(\lambda_{m}\right)\right\|_{\infty} \int_{H \backslash E_{\delta}\left(z_{j}\right)} \frac{z_{j n}^{k}}{\left|z-\bar{z}_{j}\right|^{n+k}} d z \\
& =C \delta^{-n}\left\|\left(\lambda_{m}\right)\right\|_{\infty} \int_{H \backslash E_{\delta}\left(z_{0}\right)} \frac{d z}{\left|z-\bar{z}_{0}\right|^{n+k}}
\end{aligned}
$$

for all $j$. Here, $C$ is a constant depending only on $n$ and $k$. Now, as in the proof of Theorem 5.5, we conclude that the operator $\widetilde{T}_{k} \widetilde{Q}$ is invertible for all $\delta$ sufficiently close to 1 , as desired. The proof is complete.

We now close the paper by giving a distance estimate from a given function $u \in \widetilde{\mathcal{B}}$ to the space $\widetilde{\mathcal{B}}_{0}$ as an application of Theorem 5.7. In the following $\operatorname{dist}\left(u, \widetilde{\mathcal{B}}_{0}\right)$ denotes the distance from $u$ to $\widetilde{\mathcal{B}}_{0}$ and the limsup is taken as $z \rightarrow \partial H \cup\{\infty\}$.

Proposition 5.8. Let $k \geq 1$ be an integer. Then

$$
\operatorname{dist}\left(u, \widetilde{\mathcal{B}}_{0}\right) \approx \lim \sup z_{n}^{k}\left|D^{k} u(z)\right|
$$

as $u$ ranges over $\tilde{\mathcal{B}}$.

Proof. Let $u \in \tilde{\mathcal{B}}$. It follows from (2.12) that

$$
\limsup z_{n}^{k}\left|D^{k} u(z)\right| \leq C \operatorname{dist}\left(u, \widetilde{\mathcal{B}}_{0}\right)
$$

To prove the other inequality, fix a $\delta$-lattice $\left\{z_{m}\right\}$ for which Proposition 4.12 holds. Let $j$ be a given positive integer. Choose inductively a 
subsequence $\left\{z_{j_{k}}\right\}$ of $\left\{z_{m}\right\}_{m=j+1}^{\infty}$ which is sufficiently separated so that $\left\{z_{j_{k}}\right\}$ is a $\widetilde{\mathcal{B}}_{0}$-interpolating sequence of order $k$ by Theorem 5.7. Adding finitely many points, one can find that the sequence $z_{1}, z_{2}, \cdots, z_{j}, z_{j_{1}}, z_{j_{2}}, \cdots$ is still a $\widetilde{\mathcal{B}}_{0}$-interpolating sequence of order $k$. Thus, there exists a function $g \in \widetilde{\mathcal{B}}_{0}$ such that $D^{k} g\left(z_{m}\right)=D^{k} u\left(z_{m}\right)$ for $1 \leq m \leq j$ and $D^{k} g\left(z_{m}\right)=0$ for all $m=j_{1}, j_{2}, \cdots$. Now, by Proposition 4.12 ,

$$
\|u-g\|_{\mathcal{B}} \leq C \sup _{m \geq j+1} z_{m n}^{k}\left|D^{k} u\left(z_{m}\right)-D^{k} g\left(z_{m}\right)\right| \text {. }
$$

Since $g \in \widetilde{\mathcal{B}}_{0}$ and $z_{m} \rightarrow \partial H \cup\{\infty\}$, taking the limit $j \rightarrow \infty$, one obtains

$$
\operatorname{dist}\left(u, \widetilde{\mathcal{B}}_{0}\right) \leq C \lim \sup z_{n}^{k}\left|D^{k} u(z)\right|
$$

as desired.

\section{REFERENCES}

[1] E. Amar, Suites d'interpolation pour les classes de Bergman de la boule du polydisque de $\mathbb{C}^{n}$, Canadian J. Math. 30(1978), 711-737.

[2] K. R. M. Attle, Interpolating sequences for the derivatives of Bloch functions, Glasgow Math. J. 34(1992), 35-41.

[3] S. Axler, P. Bourdon and W. Ramey, Harmonic Function Theory, Springer-Verlag, New York, 1992.

[4] B. R. Choe and K. Rim, Fractional derivatives of Bloch functions, growth rate, and interpolation, Acta Math. Hungarica 72(1-2)(1996), 67-86.

[5] R. R. Coifman and R. Rochberg, Representation theorems for holomorphic and harmonic functions in $L^{p}$, Astérisque 77(1980), 11-66.

[6] J. B. Garnett, Bounded analytic functions, Academic, New York, 1981.

[7] D. H. Luecking, Representation and duality in weighted spaces of analytic functions, Indiana Univ. Math. J. 2(1985), 319-336.

[8] W. Ramey and H. Yi, Harmonic Bergman functions on half-spaces, Trans. Amer. Math. Soc. 348(1996), 633-660.

[9] R. Rochberg, Interpolation by functions in Bergman spaces, Michigan Math. J. 29(1982), 229-236.

[10] H. Yi, Harmonic little Bloch functions on half-spaces, Math. Japonica 47(1)(1998), 21-28.

Boo Rim Choe

Department of Mathematics

Korea University

Seoul 136-701, Korea

choebr@semi.korea.ac.kr 
HeungSu Yi

Department of Mathematics

Research Institute of Basic Sciences

Kwangwoon University

Seoul 139-701, Korea

hsyi@euler.kwangwoon.ac.kr 